\title{
A DECOMPOSITION FOR COMBINATORIAL GEOMETRIES
}

\author{
BY
}

\section{THOMAS H. BRYLAWSKI}

ABSTRACT. A construction based on work by Tutte and Grothendieck is applied to a decomposition on combinatorial pregeometries in order to study an important class of invariants. The properties of this Tutte decomposition of a pregeometry into a subgeometry $G \backslash e$ and contraction $G / e$ is explored in a categorically integrated view using factored strong maps. After showing that direct sum decomposition distributes over the Tutte decomposition we construct a universal pair $(R, t)$ where $R$ is a free commutative ring with two generators corresponding to a loop and an isthmus; and $t$, the Tutte polynomial assigns a ring element to each pregeometry. Evaluations of $t(G)$ give the Möbius function, characteristic polynomial, Crapo invariant, and numbers of subsets, bases, spanning and independent sets of $G$ and its Whitney dual. For geometries a similar decomposition gives the same information as the chromatic polynomial throwing new light on the critical problem.

A basis is found for all linear identities involving Tutte polynomial coefficients. In certain cases including Hartmanis partitions one can recover all the Whitney numbers of the associated geometric lattice $L(G)$ from $t(G)$ and conversely.

Examples and counterexamples show that duals, minors, connected pregeometries, series-parallel networks, free geometries (on which many invariants achieve their upper bounds), and lower distributive pregeometries are all characterized by their polynomials. However, inequivalence, Whitney numbers, and representability are not always invariant.

Applying the decomposition to chain groups we generalize the classical twocolor theorem for graphs to show when a geometry can be imbedded in binary affine space. The decomposition proves useful also for graphical pregeometries and for unimodular (orientable) pregeometries in the counting of cycles and coboundaries.

1. Introduction. The present work is born from the application to combinatorial theory of some techniques that have been successfully used in the field of commutative algebra and algebraic topology [4]. In this introduction we shall attempt to out line the motivation, as well as the main results that we have obtained and possible future areas of exploration suggested by the se results.

Our basic starting point is the notion of a combinatorial geometry or more generally a combinatorial pregeometry (matroid) for whose motivation we refer to [10]

Received by the editors September 28, 1971.

AMS 1969 subject classifications. Primary 0527, 0535, 5060; Secondary 0545, 0630, 0635, 0640, 5020, 5030, 5530 . 
or [20]. Some of the basic definitions and elementary propositions from combinatorial geometries which we will need are summarized in the next section. Briefly, the concept of the combinatorial geometry abstracts the notion of linear dependence of sets of points in projective or affine space. As first pointed out by Whitney, this abstraction acts as a powerful unifying concept in a variety of combinatorial structures including graphs, totally unimodular matrices, and extremal combinatorial problems.

The study of such abstract projective configurations is only at its beginning. After the basic work of Whitney and Birkhoff, and some isolated contributions by Dilworth and Rado, the main results of the theory are due to Tutte, and pertain to the representation of abstractly given combinatorial geometries as concrete sets of points in projective space [19].

It would appear at first that the notions of linear algebra apply to the study of combinatorial geometries. It turns out, however, outside of certain very obvious and rather simple results, such as the notions of linear independence and bases and the pertaining result that all bases have the same number of elements etc., very little of linear algebra can be carried to arbitrary pregeometries without some a priori knowledge of a projective space into which the geometry can be embedded.

In a more general context, the study of commutative algebras and modules has led to a characterization of the trace of a matrix by the well-known work of Grothendieck. At present, one associates with every Abelian category a Grothendieck group (and sometimes a Grothendieck ring), which possesses all the information which is contained in the classical matrix case in the notion of trace and determinant.

This is precisely our starting point: by taking an abstract point of view, we carry over to combinatorial pregeometries constructions which resemble the Grothend ieck group and ring [4]. We add at the outset however that this construction was first intuited in a profound paper by W. Tutte written in 1947 [18]; therefore, credit for the idea of a Grothendieck group and ring should be shared with Tutte.

Motivated by the way the classical Grothendieck group arises from the invariance of the trace of a linear operator on certain vector space decompositions, we are led to explore two decompositions which have as an invariant not only the classical characteristic polynomial but also another generating function which may be evaluated for any pregeometry to give its number of subsets, bases, independent sets, and spanning sets.

These two decompositions-direct sum and another reminiscent of subobjectquotient object decomposition-are compatible in a certain sense resembling the distributive compatibility of ring operations. It is exactly this compatibilty in addition to the uniqueness of these two decompositions which allows us to construct a free commutative ring called the Tutte-Grothendieck ring and canonical map called the 
Tutte polynomial which composes with ring homomorphisms to give a 1-1correspondence between such homomorphisms and all functions which, like the characteristic polynomial, are invariant for the above decompositions. One makes this construction since it allows us to compute the Tutte polynomial which then serves as a universal invariant for the decomposition as well as to characterize all such invariant functions and to let knowledge of one invariant give information about another.

In $\$ 3$ we show that the category of combinatorial pregeometries has the requisite properties for the construction of a Tutte-Grothendieck ring. Our main result here is that this ring is isomorphic to a polynomial ring over the integers in two variables. When geometries are considered instead of pregeometries, one obtains a ring in one variable, which embodies all the information contained in the classical characteristic polynomial. Finally, one may consider pregeometries with basepoints and in this case a polynomial in four variables results. Naturally, constructions associated with more general pairs $(G, H)$ suggest themselves and we will study them in a future paper.

In $\$ 4$ we study those invariants which can be obtained by evaluating the Tutte polynomial of a pregeometry at various values of the two variables. It is remarkable that several classical combinatorial invariants can be obtained very easily by such evaluations. A table is given at the end of the section. These include the Möbius function and an invariant recently discovered by Crapo as well as the ones alluded to previously. Our basic result allows us to obtain these and all such invariants as evaluations of the Tutte polynomial. By viewing Whitney duality and upper lattice truncation as functors in the decomposition category, it is shown that the Tutte polynomial also allows one to compute the chromatic polynomial of a truncated pregeometry as well as all the above invariants for the Whitney dual. In $\$ 5$ we use the Higgs lift construction to prove that Tutte decompositions correspond to factored bijective unit rank decreasing strong maps thereby correlating our constructions with the categorical description of geometries in [10].

In $\$ 6$ we make a detailed study of the polynomial associated with every pregeometry in the Tutte-Grothendieck ring, which turns out to coincide with the Tutte polynomial studied by Tutte in the case of graphs [18] and by Crapo generally [8]. We obtain a complete set of linear identities which are satisfied by all but a finite number of Tutte polynomials. We also relate the coefficients of the Tutte polynomials with the various classes of closed sets or flats of combinatorial geometries. Perhaps the most interesting result in this connection is the inequality we obtain relating the Tutte polynomial of a connected geometry with the Tutte polynomial of any of its minors, showing that the coefficients are always decreasing.

As examples of our theories in $\$ 7$ we consider several classes of geometries which can be completely characterized and fruitfully studied in terms of their Tutte polynomials. They include series-parallel networks and partitions of type $n$. These 
latter partitions play an important role as not only are they conjectured to predominate in an enumeration of $n$-point pregeometries, but also they are exactly those pregeometries whose Tutte coefficients give and are derivable from a set of doubly indexed Whitney numbers. We conjecture that the Tutte polynomial of a pregeometry $G$ can be factored in the extended Tutte-Grothendieck ring if and only if $G$ is separable. In light of Stanley's results about factorization of the characteristic polynomial relative to a modular flat $([16]$ or [3]) it becomes an interesting open question as to whether the Tutte polynomial of a connected (nonseparable) pregeometry is always prime.

In $\$ 8$ we also show by simple counterexamples that the Tutte polynomial not only does not characterize a geometry up to isomorphism, but also cannot always give information about representability and Whitney numbers.

In $\$ \$ 9-12$ we introduce the notion of hereditary classes of pregeometries as subdecompositions, and show that for certain of these hereditary classes special invariants can be obtained from the Tutte-Grothendieck ring. Perhaps the most remarkable examples are the relationships with the critical problem, as described by Crapo and Rota, and with the problem of coloring a graph. In this connection our main new result is a generalization of a classical two-color theorem to arbitrary binary pregeometries. An application of these methods of decomposition give Cayley's formula for the number of trees in a complete graph [5].

We conclude with a brief survey of possible application to unimodular pregeometries, which barely touches the subject and which we hope to take up in later publications.

In closing, we shall not hide the fact that our main motivation for study of the Tutte-Grothendieck ring has been and is the critical problem, which is the natural generalization of the problem of coloring a graph to a wider class of geometries. We hope that this work will begin a new trend in the study of the coloring problem, lifting it away from the classical purely graphical methods in which it has been enmeshed in the past, and bring it into the mainstream of contemporary algebra and combinatorial theory.

We would especially like to thank Professor Gian-Carlo Rota who first suggested that Tutte's techniques could be fruitfully generalized to pregeometries [15] as well as for his many suggestions and discussions on these methods. Also we are indebted to Professors Henry Crapo, Ladnor Geissinger, Curtis Greene, Robert Norman and the referee for the ir valuable advice.

2. Basic definitions. This section surveys the relevant notions of the underlying category for our work, $\underline{G}$, the category of finite combinatorial pregeometries and strong maps discussed in Crapo and Rota [10]. We then define the basic concepts in the theory of decompositions as explored in [4]. The reader is advised to read these basic definitions rapidly and refer to them as they come up in the paper. 
A finite pregeometry or matroid, $G$, is a finite set of points with a closure operator $J_{G}(\cdot)$ or satisfying the exchange property: For any points $p, q \in G$ and any subset $P \subseteq G$, if $p \in \overline{P \cup\{q\}}$ but $p \notin \bar{P}$, then $q \in \overline{P \cup\{p\}}$. When confusion might arise we denote by $\{G\}$ the points of $G$ (without the closure structure). A geometry is a pregeometry in which the empty set and each point is closed. The lattice, $L$, of closed sets or flats of a pregeometry is called a geometric lattice and is characterized as a finite, semimodular, point lattice. Flats covering 0 in $L$ are called atoms, and flats covered by 1 are called coatoms or copoints. A bond $B$ of $G$ is the set complement of a copoint. In such lattices, each lattice element $x$ is the supremum of atoms representing closures of points and each has a welldefined rank, $r(x)$, equal to the length of any maximal chain from the 0 element (representing the closure of the empty set) to $x$. The semimodular law for $L$ states that for all $x, y \in L, r(x)+r(y) \geq r(x \wedge y)+r(x \vee y)$. Flats $x$ and $y$ form a modular pair if the latter inequality is an equality; $x$ is a modular flat if it forms a modular pair with every other flat. $r(A)$, the rank of a set of points $A \subseteq G$, is defined as $r(\bar{A})$ in the associated geometric lattice. Hence, $r(G)$, the rank of the pregeometry is $r(1)$ in the lattice.

For any subset $A$, the cardinality of $A,|A|$ denotes the number of points it contains, the corank of $A$ is the nonnegative integer $r(G)-r(A)$, and the nullity of $A$ is the nonnegative integer $|A|-r(A)$. A set of points $A \subseteq G$ represents a spanning set for $G$ if $\bar{A}=G$. A set of points, $A$, is independent if $r(A)=|A|$. Otherwise, $r(A)<|A|$ and $A$ is dependent. An independent spanning set is called a basis.

A strong map from a pregeometry $G$ into $H$ is a function $f:\{G\} \cup\{0\} \rightarrow\{H\} \cup$ $\{0\}$ (where " 0 " stands for the empty set in $G$ and $H$ respectively) such that $f(0)=$ 0 and the inverse image of any closed set in $H$ is closed in $G$. Pregeometries $G$ and $H$ are isomorpbic denoted $G \simeq H$ if there is a 1-1 correspondence, $f$, between the points of $G$ and $H$ and the closed sets of $G$ and $H$ such that for any point $p$ and closed set $K, p \in K$ iff $f(p) \in f(K)$. An isomorphism class or equivalence class of pregeometries denoted $[G]$ is the class of all pregeometries isomorphic to $G$.

A pregeometry on the point set $\{G\}$ can be uniquely determined by $\underline{C}(G)$, the family of minimal dependent sets or circuits of $G$. A family $\underline{F}$ of subsets is the circuit set for some pregeometry if no subset in $E$ properly contains another and the subsets satisfy the circuit elimination property $C^{*}$ : If $C_{1}$ and $C_{2}$ are two distinct elements of $\underline{F}$ and $e \in C_{1} \cap C_{2}$ then the set difference $\left(C_{1} \cup C_{2}\right) \backslash\{e\}$ is dependent and contains an element $C_{3} \in \underline{F}$.

$G$ may also be uniquely determined from its set of bases, $\underline{B}(G)$. A family $\underline{F}$ of incomparable subsets is the set of bases for some pregeometry if $\underline{F}$ satisfies the basis exchange axiom $\underline{B}^{*}$ : For all $B_{1}$ and $B_{2}$ in $\underline{F}$ and $p \in B_{1}$ there exists $q \in B_{2}$ such that $\left(B_{1} \backslash\{p\}\right) \cup\{q\}$ is also in $\underline{F}$. 
The (Whitney) dual of $G, \tilde{G}$, is the unique pregeometry on the same point set with a set of bases consisting of base complements of $G$. Hence $B \in \underline{B}(\tilde{G})$ iff $\{G\} \backslash B \in \underline{B}(G)$. A pregeometry is self-dual if $G \simeq \tilde{G}$.

$G$ is the direct sum of two pregeometries: $G_{1} \oplus G_{2}$ if the points of $G,\{G\}$; and circuits of $G, \underline{C}(G)$, are the disjoint union $\left\{G_{1}\right\} \cup\left\{G_{2}\right\}$ and $\underline{C}\left(G_{1}\right) \cup \underline{C}\left(G_{2}\right)$ respectively (equivalently $\underline{B}(G)$ is the set cartesian product $\underline{B}\left(G_{1}\right) \times \underline{B}\left(G_{2}\right)$ ). $G_{1}$ is then said to be a direct sum factor of $G$, and $G$ is termed separable with the flats $G_{1}$ and $G_{2}$ in $L$ as separators. If no such nontrivial direct sum decomposition exists, any two distinct points of $G$ are contained in a circuit and $G$ is termed connected. A one point direct sum factor, $p$, is an istbmus if it is no circuits of $G$ and a loop if it is itself a circuit. A point is a nonfactor if it is neither a loop nor an isthmus. A pre-Boolean algebra $B_{n m}$ is a pregeometry which is the direct sum of $n$ isthmuses and $m$ loops. A Boolean algebra $B_{n}$ is a geometry which is the direct sum of $n$ isthmuses.

If $p \in G$ we define two derived pregeometries on the point set $\{G\} \backslash\{p\}:$ the deletion, $G \backslash p$; and the contraction, $G / p$. If $A \subseteq\{G\} \backslash\{p\}$, and $\bar{A}$ denotes its closure in $G$ : then the closure on $A$ in $G \backslash p$ is defined as $\bar{A} \backslash\{p\}$ while its clo-

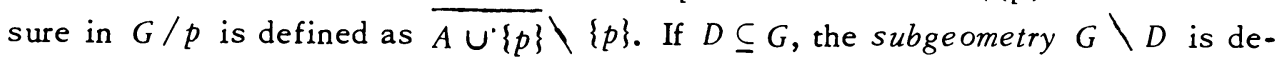
fined as a sequence of deletions by points in $D$. Similarly we define the contraction $G / D$ as a sequence of contractions. An arbitrary sequence of contractions and deletions is called a minor.

An invariant is a function $f$ defined on the class of all pregeometries such that $f(G)=f(H)$ if $G \simeq H$. Examples of invariants used in this paper include $\underline{c}(G)$, the complexity or number of bases of $G ; \underline{I}(G)$, the number of independent sets; the subgeometry generating function $S(G)=\Sigma_{i, j} a_{i j} u^{i} v^{j}$ where $a_{i j}$ is the number of subgeometries (subsets) of $G$ with corank $i$ and nullity $j$; and $\mu(G)$, the Möbius function which is defined as $\mu(0,1)$ evaluated on the geometric lattice $L$ associated with $G$, where for $x \leq y, \mu(x, y)$ is given by the recursion: $\mu(x, x)=1, \mu(x, y)=$ $-\Sigma_{x \leq z<y} \mu(x, z)$. Two other invariants evaluated on $L$ are the characteristic polynomial, $\chi(G, \lambda)=\chi(G)=\Sigma_{x \in L} \mu(0, x) \lambda^{r(1)-r(x)}$; and $\beta(G)$, the Crapo invariant which is explored in [5] with distinguishing properties: $\beta(G)=\beta(G \backslash e)+\beta(G / e)$ if $e \in G$ is neither an isthmus nor a loop, $\beta(G) \geq 0$, and $\beta(G)=0$ iff $G$ is separable.

A decomposition $\underline{D}(S)$ of a set $S$ is a rule whereby one may decompose an element $s$ in $S$ into a (finite) multisubset (subset with repetitions) $M$ of $S$ denoted $s \leq$ M. A decomposition is unique if every $s \leq S$ can be decomposed into a unique multiset of irreducible elements (elements which cannot be further decomposed). A decomposition invariant function $f$ with domain $S$ takes values in abelian group and is such that if $s$ decomposes into a multiset $M$ then $f(s)=\Sigma_{m \in M} f(m)$. If two decompositions $\underline{D}_{1}$ and $\underline{D}_{2}$ (compatibly) decompose the same set they form a bidecomposition. This bidecomposition is distributive if (1) both $\underline{D}_{1}$ and $\underline{D}_{2}$ are 
unique; (2) any element which decomposes in $\underline{D}_{1}$ into a multiset of irreducible elements is itself irreducible (for $\underline{D}_{2}$ ); and (3) $\underline{D}_{2}$ decomposition commutes with $\underline{D}_{1}$ decomposition in a manner resembling general distributivity for addition and multiplication respectively. An element which cannot be decomposed in $\underline{D}_{1}$ is termed indecomposable and hence an element which cannot be decomposed for either decomposition is an irreducible indecomposable. A bidecomposition invariant function $f$ is a function into a commutative ring which is invariant for $\underline{D}_{2}$ and also is such that for every $\underline{D}_{1}$ decomposition of $s$ into a multiset $M^{\prime}, f(s)=$ $\Pi_{m \in M^{\prime}} f(m)$.

Associated with a decomposition is its Tutte-Grotbendieck group A which along with a function mapping $S$ into $A$ is universal for decomposition invariant functions. $A$ is isomorphic to the free abelian group on $S$ factored by the subgroup generated by elements $s-\Sigma_{m \in M} m$ for any decomposition of $s$ into $M$. Associated with a bidecomposition is its Tutte-Grotbendieck ring $R$ which is the free commutative ring on $S$ factored by the ideal generated as above by all (multiplicative) $\underline{D}_{1}$ decompositions and (additive) $\underline{D}_{2}$ decompositions. When $R$ turns out to be a free commutative ring the image of an element $s$ under the natural map $t$ from $S$ into $R$ is called the Tutte polynomial of $s$.

3. The Tutte bidecomposition.

Definition 3.1 On $S$, the set of isomorphism classes of finite pregeometries, let $\underline{D}_{1}(S)$ be direct sum decomposition and let $\underline{D}_{2}$, the Tutte decomposition, be generated by decompositions of the form $G \leq G \backslash e+G / e$ for all nonfactors $e \in G$. Then since both $\underline{D}_{1}$ and $\underline{D}_{2}$ decompose pregeometries into those of smaller cardinality, the two decompositions form a bidecomposition $\underline{T}$ called the Tutte bidecomposition.

Definition 3.2. Let $Q$ be the class of polynomials whose variables are pregeometries and for a point $p$, let $D_{p}: Q \rightarrow Q$ be the partial function whose domain is all polynomials $q$ in which each term contains exactly one factor $F_{j}$ such that $p \in F_{j}$; and for such polynomials $q=\Sigma_{j} \Pi_{i} G_{i j} F_{j}$, let $D_{p}(q)=\Sigma_{j} \Pi_{i} G_{i j} F_{j}^{\prime}$ where

$$
F_{j}^{\prime}= \begin{cases}(p)\left(F_{j} \backslash p\right) & \text { if } p \text { is an isthmus or loop, } \\ \left(F_{j} \backslash p\right)+\left(F_{j} / p\right) & \text { otherwise. }\end{cases}
$$

For any $G$ and ordering $O=\left(p_{1}, \cdots, p_{n}\right)$ on the points of $G$ let $D_{O}(G)=D_{p_{n}} \circ \ldots \circ$ $D_{p_{1}}(G)$ which is well defined since $D_{p_{i}}$ is defined for all $i$ on each term of the polynomial $D_{p_{i-1}} \circ \ldots \circ D_{p_{1}}(G)$. If $q=\Sigma \Pi G_{i j}$ and $q^{\prime}=\Sigma \Pi G_{i j}^{\prime}$, define an equivalence relation $\sim$ on $Q$ such that $q \sim q^{\prime}$ if for some ordering of the terms, the pregeometries $G_{i j}$ and $G_{i j}^{\prime}$ are isomorphic. Then $Q / \sim$ is the set of all polynomials of equivalence classes of pregeometries. Denote by $\left[D_{O}(G)\right]$ the equivalence class of the polynomial $D_{O}(G)$ and denote by $x$ and $z$ the equivalence classes of 
a loop and isthmus respectively.

Lemma 3.3. For all pregeometries $G$ and orderings $O$ and $O^{\prime}$ on its points

(a) $\left[D_{O}(G)\right]$ is a polynomial with positive integer coefficients in $z$ and $x$; and

(b) $\left[D_{O}(G)\right]=\left[D_{O^{\prime}}(G)\right]$.

Proof. (a) Using induction on the number of points of $G$, we note that a one point pregeometry is necessarily an isthmus or loop and (a) holds in this case. Assume (a) for all orderings of cardinality $n-1$ and consider $D_{O}(G)$ for $O=\left(p_{1}\right.$, $\left.\cdots, p_{n}\right)$. Then if $O^{\prime \prime}=\left(p_{2}, \cdots, p_{n}\right), D_{O}(G)=D_{O^{\prime \prime}}\left(D_{p_{1}}(G)\right)=D_{O^{\prime \prime}}\left(\left(G \backslash p_{1}\right)\left(p_{1}\right)\right)$ or $D_{O^{\prime \prime}}\left(G \backslash p_{1}+G / p_{1}\right)$. But by the hypothes is $\left[D_{O \prime \prime}\left(G \backslash p_{1}\right)\right]$ and $\left[D_{O^{\prime \prime}}\left(G / p_{1}\right)\right]$ are both positive polynomials in $z$ and $x$ and the result follows for $\left[D_{O}(G)\right]$ by multiplication by $z$ or $x$ or polynomial addition respectively.

(b). We can effect any reordering by interchanging consecutive points so we need only show that $D_{O}(G)=D_{O^{\prime}}(G)$ for $O=\left(p_{1}, \cdots, p_{k}, p_{k+1}, \cdots, p_{n}\right)$ and $O^{\prime}=$ $\left(p_{1}, \cdots, p_{k+1}, p_{k}, \cdots, p_{n}\right)$. By the definition of the operator $D_{O}$, we need only check that $D_{p_{k}} \circ D_{p_{k+1}}\left(G_{i j}\right)=D_{p_{k+1}} \circ D_{p_{k}}\left(G_{i j}\right)$ on the relevant variables $G_{i j}$ of $D_{p_{k-1}} \circ \ldots \circ D_{p_{1}}(G)$.

Let $G_{i j}$ be any pregeometry containing $p_{k}$ and $p_{k+1}$. There are three cases to consider:

Case I. If $p_{k}$ is a nonfactor of $G_{i j}$, and $p_{k+1}$ is a nonfactor of both pregeometries $G_{i j} \backslash p_{k}$ and $G_{i j} / p_{k}$; then neither $p_{k}$ nor $p_{k+1}$ is an isthmus or loop of $G_{i j}$, and $\left\{p_{k}, p_{k+1}\right\}$ is not a two point circuit in either $G_{i j}$ or $\tilde{G}_{i j}$. Hence, $p_{k+1}$ is a nonfactor of $G_{i j}$ and $p_{k}$ is a nonfactor of both $G_{i j} \backslash p_{k+1}$ and $G_{i j} / p_{k+1}$. Considering the set of bases we have $\left(G_{i j} \backslash p_{k}\right) \backslash p_{k+1}=\left(G_{i j} \backslash p_{k+1}\right) \backslash p_{k} ;\left(G_{i j} \backslash p_{k}\right) / p_{k+1}=$ $\left(G_{i j} / p_{k+1}\right) \backslash p_{k} ;\left(G_{i j} / p_{k}\right) / p_{k+1}=\left(G_{i j} / p_{k+1}\right) / p_{k} ;$ and $\left.\left(G_{i j} / p_{k}\right) \backslash p_{k+1}=\left(G_{i j}\right) p_{k+1}\right) / p_{k}$. For example, both sides of the last identity define the pregeometry $P$ on the point set $\left\{G_{i j} \backslash\left(p_{k} \cup p_{k+1}\right)\right\}$ with bases those subsets of $P$ which when adjoined with $p_{k}$ form a basis for $G_{i j}$.

Case II. If $p_{k}$ is a loop (isthmus) of $G_{i j}$, then it is a loop (isthmus) of $G_{i j} \backslash p_{k+1}$ and $G_{i j} / p_{k+1}$. In any event,

$$
\left(\left(D_{p_{k+1}}\left(G_{i j}\right)\right) \backslash p_{k}\right)\left(p_{k}\right)=D_{p_{k+1}}\left(\left(G_{i j} \backslash p_{k}\right)\left(p_{k}\right)\right) .
$$

Case III. If $p_{k}$ and $p_{k+1}$ are both nonfactors of $G_{i j}$ but $p_{k+1}$ is a loop of $G_{i j} / p_{k}\left(p_{k+1}\right.$ is an isthmus of $\left.G_{i j \sim} \backslash p_{k}\right)$, then $\left\{p_{k+1}, p_{k}\right\}$ forms a circuit in $G_{i j}$ (in $\left.G_{i j}\right)$. But this means that in $G_{i j}\left(G_{i j}\right), \bar{p}_{k}=\bar{p}_{k+1}$ and there is a strong map automorphism of $G_{i j}$ which interchanges $p_{k}$ and $p_{k+1}$. Clearly then $\left[D_{O}(G)\right]=$ $\left[D_{O^{\prime}}(G)\right]$.

Since these are all the possible cases, we may denote $\left[D_{O}(G)\right]$ unambiguously by $D(G)$, the Tutte polynomial of $G$.

Example 3.4. We illustrate the computation of a Tutte polynomial: $D_{O}\left(P_{4}^{\prime}\right)$ 
where $P_{4}^{\prime}$ is a planar pregeometry of seven points, two in the same atom, whose geometric lattice is the lattice of partitions of a four element set. It is decomposed relative to the ordering $O=(a, b, c, d, e, f, g)$. Points and lines represent closed

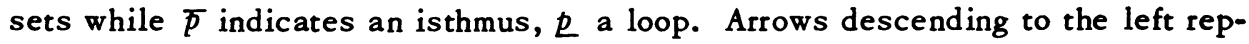
resent deletions; those to the right represent contractions; and vertical arrows show factorization. Note that in the third row two isomorphic pregeometries are identified to simplify the calculation.

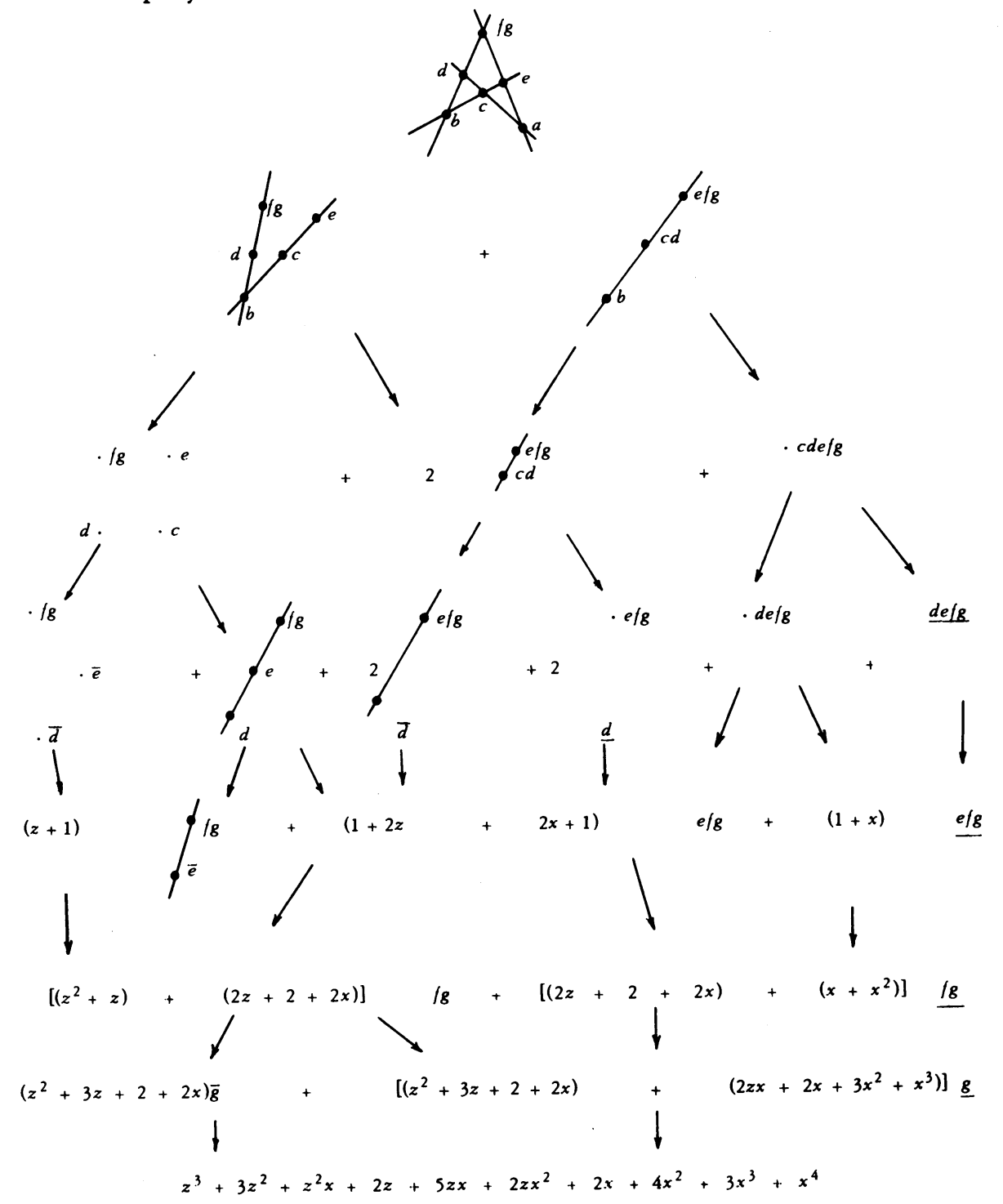

Theorem 3.5. The Tutte bidecomposition is distributive (these terms are defined in \$2) and the equivalence classes of an istbmus and a loop are the irreducible indecomposables. 
Proof. The decomposition $\underline{D}_{1}$ (direct sum decomposition) is unique with connected pregeometries as the indecomposables [10]. The irreducible elements of $\underline{D}_{2}$ are clearly pre-Boolean algebras (those pregeometries made up of entirely isthmuses and loops: $\left.B_{n m}=\bigoplus_{i=1}^{n} z \oplus \bigoplus_{j=1}^{m} x\right)$. The irreducible indecomposables are then either $x$ or $z$ and if an element has a $\underline{D}_{1}$ decomposition into these elements it is of the form $B_{n m}$ and is $\left(\underline{D}_{2}\right)$ indecomposable. Further, $\underline{D}_{2}(S)$ is a unique decomposition since we may sharpen the proof of $(3.3)$ to show that $\left[D_{O}(G)\right]$ is invariant under rearrangements in the orderings of the partial terms of the decomposition. (For example, $D_{O}(G)=D_{p_{n}} \circ \ldots \circ D_{p_{2}}\left(\left(G \backslash p_{1}\right)+\left(G / p_{1}\right)\right)=D_{p_{n}} \circ \ldots \circ$ $D_{p_{2}}\left(G \backslash p_{1}\right)+D_{p_{n}} \circ \ldots \circ D_{p_{4}} \circ D_{p_{2}} \circ D_{p_{3}}\left(G / p_{1}\right)$.) But $\underline{D}_{2}$ decomposition corresponds to partial rearrangement in which at all stages of the decomposition points which are neither isthmuses nor loops precede points which are. So as in the proof of (3.3) full $\underline{D}_{2}$-decomposition is unique and equals the Tutte polynomial $D(G)$ with $z^{n} x^{m}$ replaced by $B_{n m}$.

We will be done when we show distributivity for a basic decomposition $G=$ $G_{1} \oplus G_{2}$ of $\underline{D}_{1}$ and a basic decomposition $G_{1} \leq\left(G_{1} \backslash e\right)+\left(G_{1} / e\right)$ of $\underline{D}_{2}$. But if $e$ is a nonfactor of $G_{1}$ then it is a nonfactor of $G=G_{1} \oplus G_{2}$ since direct sum decomposition is unique, so $\underline{D}_{2}$ contains the decomposition $G \leq(G \backslash e)+(G / e)$. Also, $\underline{D}_{1}$ contains the decompositions $G \backslash e=\left(G_{1} \backslash e \oplus G_{2}\right.$ and $G / e=\left(G_{1} / e\right) \oplus G_{2}$ since the closure operator of a direct sum works componentwise, and hence for all $A_{1} \subseteq$ $\left\{G_{1}\right\} \backslash\{e\}$ and $A_{2} \subseteq\left\{G_{2}\right\}$,

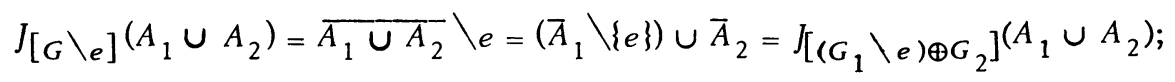

and

$$
\begin{aligned}
\left.J_{[G / e}\right]^{\left(A_{1} \cup A_{2}\right)} & =\overline{A_{1} \cup A_{2} \cup\{e} \backslash\{e\} \\
& =\left(\overline{\left.A_{1} \cup\{e\} \backslash\{e\}\right) \cup \bar{A}_{2}=J\left[\left(G_{1} / e\right) \oplus G_{2}\right]}\left(A_{1} \cup A_{2}\right) .\right.
\end{aligned}
$$

Theorem 3.6. There is a Tutte-Grothendieck ring $(R, t)$ for pregeometries associated with the Tutte bidecomposition, $T$. It is isomorphic to $P[z, x]$, the ring of polynomials in two variables without constant term over the integers (where the variable $z$ represents the coset of an istbmus and $x$ represents a loop).

Further, if $S$ is the set of isomorphism classes of pregeometries and $t: S \rightarrow R$ sends a pregeometry to its coset representation in $R$, then $t(G)=D(G)$, the Tutte polynomial of $G$ defined in (3.3).

Proof. The first part of the theorem follows from (3.5) and the results in [4] where it is shown that the universal Tutte-Grothendieck ring $T=F[S] / I$ for a distributive bidecomposition is isomorphic to a free commutative ring with basis the irreducible indecomposable elements of the bidecomposition. It is further shown that the image of the universal invariant $t$ which maps a pregeometry $G$ into the 
Tutte-Grothendieck ring is equal to the polynomial corresponding to G's (unique) decomposition first into (the additively written multiset of) $D_{2}$-indecomposable elements and then for each indecomposable its respective decomposition in $D_{1}$ into (its multiplicatively written multiset of) indecomposable irreducible elements.

The second paragraph of the theorem is a consequence of the fact that the point decomposition operator $D_{p_{i}}$ corresponds to a decomposition of $\underline{T}$ and in the free ring $F[S], G \equiv D(G)(\bmod I)$ since the ideal $I$ is generated by all identities which decompose $G$ into $D(G)$.

We will henceforth refer to the above ring for pregeometries as the TutteGrotbendieck ring. We also have (for pregeometries) the Tutte-Grotbendieck group associated with the Tutte decomposition $\underline{D}_{2}$ of $\underline{T}$.

Corollary 3.7. The Tutte-Grothendieck group $\left(A, t_{A}\right)$ is isomorphic to the free abelian group generated by pre-Boolean algebras. In addition $t_{A}(G)=$ $\left.D(G)\right|_{z^{n} x^{m} \rightarrow B_{n m}}$.

Proof. A theorem in [4] states that $A$ is group isomorphic to $R$ if $\underline{D}_{1}$ decomposition is bijective between irreducible elements of $\underline{D}_{2}$ and multisets of irreducible indecomposable elements. The corollary then follows from (3.6).

Definition 3.8. Let $U$ be the functor which takes an equivalence class of pregeometries $G$ (of rank greater than 0 ) to the equivalence class of its underlying geometry $\bar{G}$ (i.e. the geometry with the same lattice of closed sets). Then the Tutte bidecomposition $\underline{T}$. induces a decomposition $\bar{T}$ on $\bar{S}$, the set of equivalence classes of geometries, called the characteristic bidecomposition. The basic decompositions of $\bar{T}$ are $G_{1} \oplus G_{2} \leq G_{1} \times G_{2}$ and $G \leq \overline{G \backslash e}+\overline{G / e}$ for all geometries $G_{1}, G_{2}$ and $G$, and each nonisthmus $e \in G$.

Theorem 3.9. On the set $\bar{S}$ of nonempty geometries, the characteristic bidecomposition is distributive and its associated ring $(\bar{R}, \bar{t})$ called the characteristic ring is isomorphic to $P[z]$, the ring of polynomials in one variable over the integers. If $t(G)$ is the Tutte polynomial of $G$, then $\bar{t}(G)=\left.t(G)\right|_{x=0}$.

Proof. The pair $(\bar{R}, \bar{t} \circ U)$ is $\underline{T}$-invariant, where $\bar{R}$ is the characteristic ring and $\bar{t} \circ U$ sends a pregeometry $G$ to the image in $\bar{R}$ under $\bar{t}$ of $\bar{G}$, its associated geometry (or 0 if $\bar{G}$ is empty). Hence by the universality of $R$ and (3.6) there is a ring homomorphism $b: R \rightarrow \bar{R}$ where $b(z)=z$ and $b(x)=0$. since an isthmus is a geometry and the empty geometry underlies a loop. The rest of the theorem now follows.

Corollary 3.10. The additively written decompositions of $\bar{T}$ form a unique decomposition called the characteristic decomposition. The associated group $\bar{A}$ is the free abelian group generated by Boolean algebras $B_{n}$, while $\bar{t}_{A}=\left.\bar{t}_{R}\right|_{z{ }^{n} \rightarrow B_{n}}$. 
Proof. Apply the arguments of (3.7) to (3.9).

Proposition 3.11. Let $f$ be a $\underline{T}$-invariant with associated ring bomomorphism $b$. Then a necessary and sufficient condition for $f$ when restricted to geometries to be a $\bar{T}$-invariant is that $f(x)=0$ (or equivalently $b(x)=0$ ). Similarly a function which is group invariant for the Tutte decomposition is invariant for the characteristic decomposition iff the associated group homomorphism $b$ bas its support on Boolean algebras.

Conversely a function $\bar{g}$ which is a $\bar{T}$-invariant can be extended uniquely to a T-invariant $g$ by defining

$$
g(G)= \begin{cases}0 & \text { if } G \text { bas any loops, } \\ \bar{g}(G) & \text { otherwise, }\end{cases}
$$

with similar extensions for group invariants.

Proof. We will prove the proposition for ring invariants. Proofs for group invariants are entirely analogous.

If $b(x)=0$ then, since $t(G)=\left.t(G)\right|_{x=0}, f(G)=b^{\prime} \circ \bar{t}(G)$ for all geometries $G$ where $b^{\prime}$ is the restriction of $b$ to polynomials in the variable $z$. Hence by (3.9) $f$ is a $\underline{T}$-invariant.

Conversely, if $f$ is both a $\bar{T}$-invariant and a $T$-invariant on geometries, then if $z$ represents an isthmus, $x$ a loop, and $L_{3}$ the three point line: $f\left(L_{3}\right)=(f(z))^{2}+$ $f(z)=(f(z))^{2}+f(z)+f(x)$. Hence $f(x)=0$.

If $\bar{g}$ is a $\bar{T}$-invariant which is to be extended to a $\bar{T}$-invariant $g$ then by the above, $g(G)=0$ if $G$ has any loops. Otherwise, proceeding by induction on the number $n$ of points of $G$ minus the number of atoms $(|G|-|\bar{G}|)$ if $n=0$ then $G=\bar{G}$ and certainly $g(G)$ must equal $g(\bar{G})$. But if $n>0$ and a point $p$ is in the closure of another point $q$ then $G / p$ has a loop and must be equal to 0 while $\overline{G \backslash} p=\bar{G}$ and hence $g$ is an invariant if we define $g(G)=g(G \backslash p)+g(G / p)=g(\bar{G})+0$.

An application of the characteristic decomposition to the study of modular flats can be found in [3].

The category $\underline{G}^{\prime}$ of pointed pregeometries $(F, p)$ and strong maps $f:(F, p) \rightarrow$ $\left(F^{\prime}, p^{\prime}\right)$ such that $f(p)=p^{\prime}$ and $f^{-1}\left(p^{\prime}\right)=p$ was defined in [2]. Three functors were also defined from $\underline{G}^{\prime}$ to $\underline{G}$, the cateogry of pregeometries and strong maps $T((F, p))=F, T^{\prime}((F, p))=F \backslash p$, and $T^{\prime \prime}((F, p))=F / p$.

This category provides a natural setting for the study of series-parallel networks and the Tutte bidecomposition of such point pregeometries facilitates the calculation of some important network invariants as shown in [2].

Definition 3.12. Let $S^{\prime}$ be the set of equivalence classes of pregeometries $S$ and pointed pregeometries (where no pregeometry is equivalent to a pointed pregeometry). The pointed Tutte bidecomposition $T^{\prime}\left(S^{\prime}\right)$ includes the decompositions of the Tutte subbidecomposition $T(S)$ as well as basic decompositions of the form 
$(F, p) \leq((F, p) \backslash e)+((F, p) / e) \in \underline{D}_{2}^{\prime}$ and $(F, p) \oplus G \leq(F, p) \times G \in \underline{D}_{1}^{\prime}$ for all pointed pregeometries $(F, p)$ and pregeometries $G$, and for all nonfactors $e \in\{F\} \backslash\{p\}$ where $(F, p) \oplus G$ is the pointed pregeometry $(F \oplus G, p)$ and $(F, p) \backslash e((F, p) / e)$ is the pointed pregeometry $(F \backslash e, p)((F / e, p))$. A function invariant for this decomposition is termed a $T^{\prime}$-invariant. Examples of $T^{\prime}$-invariants include $f \circ T$ where $T$ is the forgetful functor which sends a pointed pregeometry $(F, p)$ to its underlying pregeometry $F$ and $f$ is a $\underline{T}$-invariant.

Theorem 3.13. The pointed Tutte bidecomposition $I^{\prime}$ is distributive and with it we may associate the pointed Tutte-Grotbendieck ring $\left(R^{\prime}, t^{\prime}\right)$ where $R^{\prime}\left[z, x, z^{\prime}, x^{\prime}\right]$ is isomorpbic to the polynomial ring over the integers in four variables corresponding to the equivalence classes of an isthmus, loop. pointed isthmus, and pointed loop respectively. Further, the pointed Tutte polynomial $t^{\prime}(F)$ equals $z^{\prime} f_{1}(z, x)+x^{\prime} f_{2}(z, x)$ for all pointed pregeometries $F$, and $t^{\prime}(G)=f(z, x)$ for all nonpointed pregeometries $G$.

Proof. These statements are all proved in [2].

The following the orem summarizes some applications of the pointed TutteGrothendieck ring to the theory of series-parallel networks. All proofs can be found in [2].

Theorem 3.14. If $(F, p)$. is a pointed pregeometry and $T$ is the forgetful functor: $T((F, p))=F$, then by universality of $R^{\prime},(t \circ T)((F, p))=z f_{1}+x f_{2}$ where the pointed Tutte polynomial of $(F, p), t^{\prime}((F, p))$, equals $z^{\prime} f_{1}+x^{\prime} f_{2}$. The Tutte polynomial also commutes with the deletion and contraction functors: $t(F \backslash p)=t(F / p)=$ $f_{1}+f_{2}$ if $p$ is a factor of $(F, p)$ while if $p$ is a nonfactor, $t(F \backslash p)=(z-1) f_{1}+f_{2}$ and $t(F / p)=f_{1}+(x-1) f_{2}$. In the category of pointed pregeometries there is a direct sum called the parallel connection, $P(G, H)$. This corresponds to the graph theoretic concept when $G$ and $H$ are graphical. In addition one may use Whitney duality to define the series connection, $S(G, H)=\widetilde{P(\widetilde{G}, H}$. If $p$ is a nonfactor of $G$ and $t^{\prime}(G)=z^{\prime} g_{1}+x^{\prime} g_{2}$ while $t(H)=z^{\prime} b_{1}+x^{\prime} b_{2}$, then

$$
t^{\prime}(S(G, H))=z^{\prime}\left[(z-1) g_{1} b_{1}+g_{1} b_{2}+g_{2} b_{1}\right]+x^{\prime} g_{2} b_{2}
$$

and

$$
t^{\prime}(P(G, H))=z^{\prime} g_{1} b_{1}+x^{\prime}\left[(x-1) g_{2} b_{2}+g_{1} b_{2}+g_{2} b_{1}\right] .
$$

4. Decomposition invariants. In this section we explore the Tutte bidecomposition $\underline{T}$ in more detail and give examples of some useful functions which are invariant on $T$.

Proposition 4.1. (a) For all pregeometries $G$ and points $e \in G$, if $A$ is a subset of $\{G\} \backslash\{e\}$, then the rank $r(A)$ of $A$ in the associated geometric lattice $L(G)$ 
is the same as the rank $r^{\prime}(A)$ computed in the lattice $L(G \backslash e)$ associated with the deletion of $e$.

(b) There is a lattice isomorphism from the lattice of the contraction $L(G / e)$ into $L(G), f:[0, G / e] \simeq[e, G]$ where for all closed sets $x$ in $G / e, f(x)=x \cup e, a$ closed set of $G$. Hence if $e \in A \subseteq G$, then $r(A)$ computed in $L(G)$ is equal to $r^{\prime \prime}(A \backslash\{e\})+1$ computed in $L(G / e)$ if $e$ is not a loop.

(c) A point $e$ of $G$ is therefore an istbmus iff $r(G)=r(\{G\} \backslash\{e\})+1=r^{\prime}(G \backslash e)$ +1 . Otherwise $r(G)=r^{\prime}(G \backslash e)$. Also $e \in G$ is a loop iff $r(G)=r^{\prime \prime}(G / e)$ and $r(G)=$ $r^{\prime \prime}(G / e)+1$ otherwise.

Proof. (a) Since for subsets $A$ of $G \backslash e$, the closure of $A$ is. $\bar{A} \backslash\{e\}$, the lattice of closed sets of $G \backslash e, L(G \backslash e)$ can be formed by taking suprema (in $G$ ) of points in $\{G\} \backslash\{e\}$. Hence on these subsets, rank is invariant in $G$ and $G \backslash e$. But $e \in G$ is an isthmus iff it is a bond iff $\{G\} \backslash\{e\}$ is a copoint of $G$.

(b) The closure operator $J$ in $G / e$ for the set $\underline{A}$ of subsets $A$ of $\{G\} \backslash\{e\}$ is $\overline{A \cup\{e} \backslash \backslash\{e\}$ and so is isomorphic to the closure operator $J^{\prime}$ in $G$ for the set $\underline{A}^{\prime}$, subsets $A^{\prime}$ of $G$ containing $e$ by the isomorphism $f(A)=A \cup\{e\}, f^{-1}\left(A^{\prime}\right)=A^{\prime} \backslash\{e\}$ in the following commutative diagram.

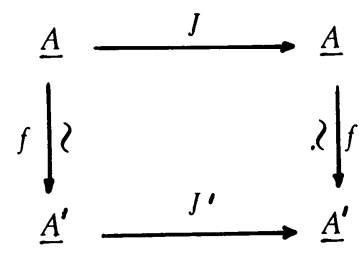

(c) A point $e \in G$ is an isthmus iff $e$ is a bond iff $\{G\} \backslash\{e\}$ is a copoint of $G$ iff $r(G)=r(\{G\} \backslash\{e\})+1=r^{\prime}(G \backslash e)+1$ (since rank is invariant in $G$ and $G \backslash e$ on subsets of $\{G\} \backslash\{e\})$. Otherwise $\overline{G \backslash e}=G$ and $r^{\prime}(G \backslash e)=r(\{G\} \backslash\{e\})=r(G)$.

The rank of $G / e$ equals by (b), the length of a maximal chain in $L(G)$ between $e$ and $G$. This chain has length $r(G)-1$ or $r(G)$ depending on whether $e$ is (in) an atom or in the closure of the empty set (i.e. a loop) respectively.

Theorem 4.2. Let $\underline{K}(G)$ denote the closed sets of $G$ and let

$$
\chi(G, \lambda)=\chi(G)=\sum_{x \in \underline{K}(G)} \mu(0, x) \lambda^{r(G)-r(x)}
$$

be the characteristic polynomial of $G$. Then the invariant defined by

$$
\begin{cases}0 & \text { if } G \text { bas a loop, } \\ (-1)^{r(G)} \chi(G) & \text { otherwise, }\end{cases}
$$

is an invariant of the Tutte bidecomposition $T$. (From now on when we apply the geometric invariant $\chi$ to pregeometries we will define $\chi(G)$ to be 0 if $G$ bas a loop.) 
Proof.

$$
\begin{aligned}
& (-1)^{r\left(G_{1} \oplus G_{2}\right)} \chi\left(G_{1} \oplus G_{2}\right) \\
& =(-1)^{r\left(G_{1} \oplus G_{2}\right)} \sum_{x \in \underline{K}\left(G_{1} \oplus G_{2}\right)} \mu(0, x) \lambda^{r\left(G_{1} \oplus G_{2}\right)-r(x)} \\
& =(-1)^{r\left(G_{1}\right)+r\left(G_{2}\right)} \sum_{x^{\prime} \in \underline{K}\left(G_{1}\right)} \sum_{x^{\prime \prime} \in \underline{K}\left(G_{2}\right)} \mu\left(0, x^{\prime} \bigvee x^{\prime \prime}\right) \lambda^{r\left(G_{1}\right)+r\left(G_{2}\right)-r\left(x^{\prime}\right)-r\left(x^{\prime \prime}\right)} \\
& =\left[(-1)^{r\left(G_{1}\right)} \sum_{x^{\prime} \in \underline{K}\left(G_{1}\right)} \mu\left(0, x^{\prime}\right) \lambda^{r\left(G_{1}\right)-r\left(x^{\prime}\right)}\right] \\
& \cdot\left[(-1)^{r\left(G_{2}\right)} \sum_{x^{\prime \prime} \in \underline{K}\left(G_{2}\right)} \mu\left(0, x^{\prime \prime}\right) \lambda^{r\left(G_{2}\right)-r\left(x^{\prime \prime}\right)}\right] \\
& =\left[(-1)^{r\left(G_{1}\right)} \chi^{\left.\left(G_{1}\right)\right]\left[(-1)^{r\left(G_{2}\right)} \chi\left(G_{2}\right)\right]}\right.
\end{aligned}
$$

since in lattice cartesian products, $\mu\left(0, x^{\prime} \vee x\right)=^{\cdot} \mu\left(0, x^{\prime}\right) \mu\left(0, x^{\prime \prime}\right)$ [14].

By (3.11) and noting that $\chi(G)$ depends only on the geometric lattice we need only show that the theorem holds for geometries. In that case using spanning sets $y$ of atoms for the subgeometry $x$ defined by all closed sets $x \in \underline{K}(G)$, we obtain by order dualizing Proposition 5.1 from [14] the formula $\mu(0, x)=\Sigma_{\bar{y}=x}(-1)|y|$. Hence if $e \in G$ is not an isthmus:

$$
(-1)^{r(G)} \chi(G)=\left.(-1)^{r(G)} \sum_{y \subsetneq \varsigma G}(-1)^{|y|}\right|_{\lambda^{r(G)-r(y)}}
$$

(since the closed sets $x \in \underline{K}(G)$ partition subsets of $G$ into spanning subsets for $x$ ). But summing separately over those subsets which do not contain $e$ and those which do, the formula for the characteristic polynomial becomes

$$
\begin{aligned}
& (-1)^{r(G)} \sum_{e \notin y}(-1)^{|y|} \lambda^{r(G)-r(y)}+\left.(-1)^{r(G)} \sum_{e \in y}(-1)^{\mid y}\right|_{\lambda^{r(G)-r(y)}} \\
& =(-1)^{r^{\prime}(G \backslash e)} \sum_{y \subseteq G \backslash l e}(-1)|y|_{\lambda} r^{r^{\prime}(G \backslash e)-r^{\prime}(y)}
\end{aligned}
$$

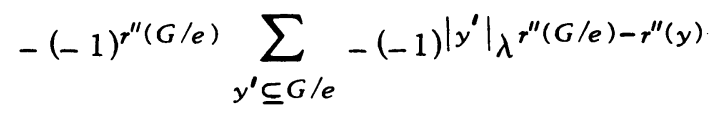

$$
\begin{aligned}
& =(-1)^{r^{\prime}(G \backslash e)} \chi(G \backslash e)+(-1)^{r^{\prime \prime}(G / e)} \chi(G / e)
\end{aligned}
$$

using the results and isomorphism $f$ of (4.1).

Corollary 4.3. For all pregeometries $G$, if the Tutte polynomial $t(G)$ equals 
$f(z, x)$, then $\chi(G)=(-1)^{r(G)} f(1-\lambda, 0)$. In particular, $\mu(G)=(-1)^{r(G)} f(1,0)$.

Proof. By (3.6) and (4.2), we need only compute $(-1)^{r(G)} \chi(G)$ for an isthmus $z$ and a loop $x$. But $(-1)^{r(z)} \chi(z)=(-1)(\lambda-1)$ while $(-1)^{r(x)} \chi(x)=0$. Hence $f(1-\lambda, 0)=(-1)^{r(G)} \chi(G)$. In particular $\mu(G)=\mu(0, G)=\left.\chi(G)\right|_{\lambda=0}=$ $\left.(-1)^{r(G)} f(1-\lambda, 0)\right|_{\lambda=0}$.

It is the above universal description of $\chi$ which allows one to show in [3] that $\chi(x)$ divides $\chi(G)$ for any geometry $G$ and modular flat $x$ of $G$.

Corollary 4.4. A function $f$ is an invariant (of botb $\underline{T}$ and) $\bar{T}$ if and only if it is an evaluation of $\chi(G)$.

Proof. By (3.11), $f$ is invariant under $\underline{T}$ and $\underline{T}$ iff $f(x)=0$ iff $f$ is an evaluation of $\left.t(G)\right|_{x=0}$ iff $f$ is an evaluation of $\left.t(G)\right|_{x=0, z=1-\lambda}$.

Theorem 4.5. The subgeometry generating function, $S(G)=\Sigma a_{i j} u^{i} v^{j}$, is a $\underline{T}$ invariant where $a_{i j}$ is the number of subgeometries of $G$ with corank $i$ and nullity $j$ (bence if $r(G)=n, a_{n-k, m-k}$ counts the number of subgeometries of $G$ with rank $k$ and cardinality $m$ ).

Proof. If $G=G_{1} \oplus G_{2}$, the rank of any subset of $G$ is the sum of the ranks of the subset when intersected with the two sets $\left\{G_{1}\right\}$ and $\left\{G_{2}\right\}$. This holds in particular for $G$, hence it holds for corank as well as cardinality and hence nullity. Thus

$$
\begin{aligned}
& S\left(G_{1} \oplus G_{2}\right)=\sum a_{i j} u^{i} v^{j}=\sum_{A \subseteq G_{1} \oplus G_{2}} u^{r\left(G_{1} \oplus G_{2}\right)-r(A)} v|A|-r(A) \\
& =\sum_{A \subseteq\left(G_{1} \cup G_{2}\right)} u^{r\left(G_{1}\right)+r\left(G_{2}\right)-r\left(A \cap G_{1}\right)-r\left(A \cap G_{2}\right)} v\left|A \cap G_{1}\right|+\left|A \cap G_{2}\right|-r\left(A \cap G_{1}\right)-r\left(A \cap G_{2}\right) \\
& =\sum_{A^{\prime} \subseteq G_{1}} u^{r\left(G_{1}\right)-r\left(A^{\prime}\right)} v_{v}\left|A^{\prime}\right|-r\left(A^{\prime}\right) \sum_{A^{\prime \prime} \subseteq G_{2}} u^{r\left(G_{2}\right)-r\left(A^{\prime \prime}\right)} v_{\nu}\left|A^{\prime \prime}\right|-r\left(A^{\prime \prime}\right) \\
& =S\left(G_{1}\right) S\left(G_{2}\right) \text {. } \\
& \text { If } e \in G \text { is neither an isthmus nor a loop }
\end{aligned}
$$

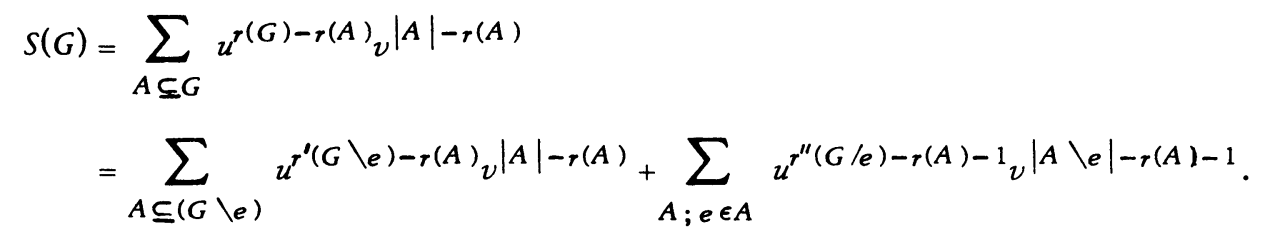

But the sinister is equal to $S(G \backslash e)$ while the isomorphisms $f^{-1}$ from $[e, G]$ to $[0, G / e]$ shows that the dexter is equal to $S(G / e)$. 
Corollary 4.6. For all pregeometries $G$, if the Tutte polynomial $t(G)$ equals $f(z, x)$, then $S(G)=f(u+1, v+1)$. In particular the number of spanning sets of $G$ equals $f(1,2)$; the number of independent sets is $f(2,1)$; the number of bases equals $f(1,1)$; and the number of subsets equals $f(2,2)$. More generally, if $n$ is the rank of $G$, the number of independent sets of cardinality $k$ is given by replacing $z^{i} x^{j}$ by $\left(\begin{array}{c}i \\ n-k\end{array}\right)$ in $t(G)$, and the number of spanning sets of cardinality $k$ by replacing $z^{i} x^{j}$ by $\left(\begin{array}{c}j \\ k-n\end{array}\right)$.

Proof. By (3.6) and (4.5) we need only compute $S(G)$ for an isthmus $z$ and a loop $x$. But $S(z)=u+1$ (both subgeometries have nullity 0 ) while $S(x)=v+1$. (both subgeometries have rank and corank 0 ). Hence $s(G)=f(u+1, v+1)$. In particular $f(1,2)=\left.S(G)\right|_{u=0, v=1}=\Sigma_{j} a_{0, j}=$ the number of subgeometries with corank 0 , i.e. the spanning sets; $f(2,1)=\left.S(G)\right|_{u=1, v=0}=\Sigma_{i} a_{i, 0}=$ the number of subgeometries with nullity 0 , i.e. the independent sets; $f(1,1)=a_{0,0}$, the bases; while $f(2,2)=\Sigma a_{i j}$, the number of subsets $(2|G|)$.

Some further applications of the previous theorems can be found in [2] where, in conjunction with (3.14), the invariants of (4.2) and (4.5) are computed across a series and a parallel connection. As a corollary to these results, it is shown that a series-parallel network (a pregeometry which contains neither a four point line nor $P_{4}$, the geometry of partitions of a four element set, as minors) is three colorable.

Certain operators on pregeometries are functors from the Tutte decomposition into itself (i.e. preserve decompositions) and lead to important invariants.

Theorem 4.7. Letting $\tilde{G}$ denote the (Whitney) dual of the pregeometry $G$, then if $\tilde{t}(G)=t(\tilde{G})$, the Tutte polynomial of the dual pregeometry, then $\tilde{t}$ is a $\underline{T}$-invariant. In addition, if $f(z, x)$ is the Tutte polynomial of $G$, then $f(x, z)$ is the Tutte polynomial of $\tilde{G}$.

Proof. If $G=G_{1} \oplus G_{2}$ then the set of bases of $G, \underline{B}(G)$ is the cartesian product $\underline{B}\left(G_{1}\right) \times \underline{B}\left(G_{2}\right)$. But for all subsets $B_{1} \subseteq G_{1}$ and $B_{2} \subseteq G_{2}: B_{1} \cup B_{2} \in \underline{B}(\tilde{G})$

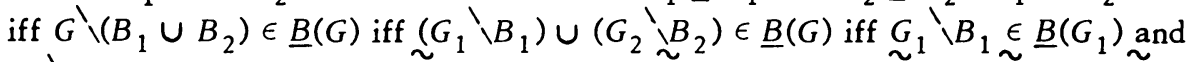
$\underline{G}_{2} \backslash B_{2} \in \underline{B}\left(G_{2}\right)$ iff $B_{1} \in \underline{B}\left(\tilde{G}_{1}\right)$ and $B_{2} \in \underline{B}\left(\tilde{G}_{2}\right)$. Hence $\underline{B}(\tilde{G})=\underline{B}\left(\tilde{G}_{1}\right) \times \underline{B}\left(\tilde{G}_{2}\right)$ and $\widetilde{G}_{1} \oplus G_{2}=\tilde{G}_{1} \oplus \tilde{G}_{2}$.

By the previous paragraph, $G=(G \backslash e) \oplus e$ iff $\tilde{G}=(\widetilde{G \backslash e}) \oplus \tilde{e}$ and so $e$ is a nonfactor in $G$ iff it is one in $\tilde{G}$; and if this is the case: $B \in \underline{B}(\tilde{G} \backslash e)$ iff $e \notin B \in$ $\underline{B}(\tilde{G})$ iff $e \in(G \backslash B) \in \underline{B}(G)$ iff $(G \backslash B) \backslash e \in \underline{B}(G / e)$ iff $B \in \underline{B}(\widetilde{G / e})$. Hence $\widetilde{G} \backslash e=$ $\widetilde{G / e}$ and dualizing, $\tilde{\sim} / e=G \backslash e$. Thus if $G$ decomposes into $G \backslash e$ and $G / e ; \tilde{G}$ decomposes into $\tilde{G} / e$ and $\tilde{G} \backslash e$. This shows that $\tilde{t}$ is a $\underline{T}$-invariant (taking values in $R$ ). The dual of an isthmus (with the isthmus as the only basis) is a loop (with the empty set as the only basis) and conversely.

Lemma 4.8. If $t(G)=\Sigma b_{i j} z^{i} x^{j}$ is the Tutte polynomial of $G$, then the mapping $t_{A}(G)$ of $G$ into the Tutte-Grothendieck group, $A$, is equal to $\Sigma b_{i j} B_{i j}$, and if $|G| \geq$ 2 , then $b_{10}=b_{01}$. 
Proof. The fact that the coefficients of $z^{i} x^{j}$ and $B_{i j}$ are the same was shown in (3.7). If $|G| \geq 2$, the only way to get a one point pregeometry which would contribute to either $b_{01}$ or $b_{10}$ is to decompose a two point pregeometry by a morphism in $\underline{D}_{2}$. But the only pregeometry of two points which is connected (hence has a point which is not a one point factor) is the two point circuit $C_{2}$ and the only decomposition available is $C_{2} \leq x+z$ (or $C_{2} \leq B_{10}+B_{01}$ ).

This linear identity involving the coefficients of the Tutte polynomial is the first in a basis for all such identities developed later (6.6).

Theorem 4.9. The Crapo "Betsy" invariant $\beta(G)=(-1)^{r(G)} \Sigma_{A \subseteq G}(-1)^{|A|} r(A)$ defined in [6] is an invariant of the Tutte decomposition where if $t_{A}(G)=$ $\Sigma b_{i j} B_{i j}, \beta(G)=b_{10}$. Further, for all $|G| \geq 2$, and for any invariant $f$ which, like $\beta$, vanishes on separable pregeometries, $f$ is a multiple of $\beta$ and by properties of $t_{A}, f(G)=f(\tilde{G})$.

Proof. That $\beta(G)=\beta(G \backslash e)+\beta(G / e)$ for all nonfactors $e$ and that $\beta(G)=0$ for all separable $G$ was proved in [6]. Thus $\beta$ is an evaluation of $t_{A}(G)$. But $\beta\left(B_{i j}\right)=0$ for all $i+j>1$ and $\beta\left(B_{10}\right)=1$ while $\beta\left(B_{01}\right)=0$. Hence $\beta(G)=b_{10}$. If $f$ is any other Tutte decomposition invariant with support on connected pregeometries, then $f\left(B_{i j}\right)=0$ for all $i+j>1$ and hence $f(G)=k_{1} b_{10}+k_{2} b_{01}$. But by (4.8), $f(G)=\left(k_{1}+k_{2}\right) b_{10}=\left(k_{1}+k_{2}\right) \beta(G)$.

Corollary 4.10. $\beta(G)$ is an invariant of the characteristic decomposition and $\beta(G)=d(\chi(G)) /\left.d \lambda\right|_{\lambda=1}$.

Proof. From (4.9) and (3.11), $\beta(G)$ is an invariant of the characteristic decomposition. In addition $\beta(G)=b_{10}=\partial(t(G)) /\left.\partial z\right|_{x=0, z=0}=d(\chi(G)) /\left.d \lambda\right|_{\lambda=1}$.

Although upper truncation is a useful operation on geometries there seems to be no nontrivial category in which it is a functor. The following proposition shows that perhaps the characteristic decomposition is the proper category in which to consider truncation.

Proposition 4.11. If $G$ is a geometry and $T_{1}(G)$ denotes the upper rank one truncation of $G$ (i.e. the geometry associated with the geometric lattice formed from $L(G)$ by removing the copoints and letting the colines become the new copoints) then $T_{1}(G)$ preserves characteristic decomposition and if $\bar{t}_{A}(G)=\Sigma b_{i} B_{i}$ then $\bar{t}_{A}\left(T_{1}(G)\right)=\Sigma_{i>0}\left(\Sigma_{j>i} b_{j}\right) B_{i}$.

Proof. If $G$ has rank one, $T_{1}(G)$ is the empty geometry. If $G$ has rank two, $T_{1}(G)$ is an isthmus. Otherwise $G$ and $T_{1}(G)$ have the same number of atoms. If $A$ and $G \backslash A$ are two nontrivial flats of $G, r(A)+r(G \backslash A)>r(G)>r(T(G)$ ). Hence $T(G)$ can have no separators and is connected. So $e \in T(G)$ is never an isthmus or loop.

By the remarks on the closed sets of $G / e$ and $G \backslash e$ in $(4.1), L(G / e) \simeq[e, G]$ in $L(G)$. Hence copoints in $G \backslash e$ are in 1-1 correspondence with copoints con- 
taining $e$ in $G$, and $L\left(\left(T_{1}(G)\right) / e\right)=L\left(T_{1}(G / e)\right)$.

Rank and cardinality of subsets of $\{G\} \backslash e$ are invariant in $G$ and $G \backslash e$. Hence $L\left(T_{1}(G \backslash e)\right)=\left(\left(T_{1}(G)\right) \backslash e\right)$ since both sides represent the lattice of closed sets of the geometry $P$ whose family of independent sets $\underline{I}(P)$ is given by $\underline{I}(G \backslash e) \backslash \underline{B}(G \backslash e)$ (the independent nonbases of $G \backslash e$ ).

$A$ theorem in [4] states that an operator $T$ preserves decompositions if and only if there is an endomorphism $b$ of $A$ which reflects the action of $T$ (i.e. $t_{A} \circ T=$ $b \circ T)$. Hence one may compute $t_{A} \circ T_{1}(G)$ if $t_{A}(G)$ is known. For the Boolean algebra $B_{n}, T_{1}\left(B_{n}\right)=C_{n}$ the $n$ point circuit. For any $e \in C_{m}, C_{m} \backslash e=B_{m-1}$ and $C_{m} / e=C_{m-1}$. Therefore $t_{A} \circ T_{1}\left(B_{n}\right)=\sum_{i=1}^{n-1} B_{i}$ and the result follows.

Table 4.12. Interpretations of some of the evaluations of the Tutte polynomial $t(G)$ are summarized below:

\begin{tabular}{|c|c|c|c|c|}
\hline & $-i$ & 0 & 1 & 2 \\
\hline & & $\begin{array}{l}(-1)^{r(G)} \chi^{(G, i+1)} \\
\text { (nowhere zero } \\
\text { coboundaries) }\end{array}$ & & \\
\hline 0 & $\begin{array}{c}(-1)^{r(\tilde{G})} \chi(\widetilde{G}, i+1) \\
\text { (nowhere zero } \\
\text { cycles) }\end{array}$ & 0 & $|\mu(\tilde{G})|$ & \\
\hline 1 & & $|\mu(G)|$ & $\begin{array}{c}\text { complexity } \\
\text { (number of } \\
\text { bases) }\end{array}$ & $\begin{array}{l}\text { number of } \\
\text { spanning } \\
\text { sets }\end{array}$ \\
\hline 2 & & & $\begin{array}{l}\text { number of } \\
\text { independent } \\
\text { sets }\end{array}$ & $\begin{array}{c}\text { number of } \\
\text { subgeometries } \\
\text { (subsets) }=2^{G}\end{array}$ \\
\hline
\end{tabular}

In addition, $\partial t(G) /\left.\partial z\right|_{z=x=0}=\beta(G)$, and the number of independent (spanning) sets of cardinality $k=\left.t(G)\right|_{z^{i} x^{j}}=\left({ }_{r(G)-k}^{i}\right)\left(=\left(\begin{array}{c}j \\ k-r(G)\end{array}\right)\right.$.

5. Decomposition and strong map factorization. In this section we examine the Tutte decomposition in the context of the category of pregeometries and strong maps. We make use of a lift construction of D. Higgs [12] in which a strong map is factored as an injection followed by a contraction.

Lemma 5.1. If $f: G^{\prime} \rightarrow G^{\prime \prime}$ is a strong map epimorphism from the geometry $G^{\prime}$ onto the geometry $G^{\prime \prime}$ then there exists a geometry $G$ and strong maps $f^{\prime}: G^{\prime} \rightarrow G$ 
and $f^{\prime \prime}: G \rightarrow G^{\prime \prime}$ such that $f^{\prime}$ is the injection of a subgeometry $G^{\prime}$ into $G, f^{\prime \prime}$ is a contraction, and $f=f^{\prime \prime} . \circ f^{\prime}$.

Regarding $G, G^{\prime}$, and $G^{\prime \prime}$ as geometric lattices then $G$ is isomorphic to a quotient of $G^{\prime} \oplus Q$ (a geometric sublattice of $G^{\prime} \oplus Q$ associated with a closure operator $-G$ ) where $Q$ is an arbitrary geometric lattice of rank $r\left(G^{\prime}\right)-r\left(G^{\prime \prime}\right)$. Denoting the elements of $G^{\prime} \oplus Q$ by $(x, y)$ where $x \in G^{\prime}$ and $y \in Q$, the interval $\left[\overline{(0,1)}^{G}, \overline{(1,1)}^{G}\right]$ in $G$ is isomorphic to $G^{\prime \prime}$ and $f^{\prime \prime}$.is contraction of $G$ by the element $\overline{(0,1)}{ }^{G}$. The injection $x \rightarrow(x, 0)$ of $G^{\prime}$ into $G^{\prime} \oplus Q$ followed by $G$-closure as a strong map onto $G$ is an injection of $G^{\prime}$ into $G$.

In the special case in which $r\left(G^{\prime}\right)-r\left(G^{\prime \prime}\right)=1$, then $G$ is the sublattice consisting of those elements $\left\{\left(x_{i}, 0\right)\right\} \cup\left\{\left(y_{i}, 1\right)\right\}$ of $G^{\prime} \oplus Q$ in which $x_{i}$ is the supremum of all elements of $G^{\prime}$ with given image and $y_{j}$ is a minimal element with given image.

Proof. A proof of this theorem can be found in [10] as well as [12].

Theorem 5.2. Two pregeometries $G^{\prime}$ and $G^{\prime \prime}$ are the Tutte decomposition of a pregeometry $G$ if and only if they differ in rank by one and there exists a 1-1 onto strong map $f$ between them. In this case the map $f$ may be viewed as the composition of injection of $G \backslash e$ into $G$ followed by contraction of $G$ onto G/e for some point $e \in G$ where $G \backslash e \simeq G^{\prime}$ and $G / e \simeq G^{\prime \prime}$. Further this $G$ is unique.

Proof. If $G<G \backslash e+G / e \in \underline{D}_{2}$ then by (4.1), $r^{\prime}(G \backslash e)=r^{\prime \prime}(G / e)+1$ while the composite of the injection $G \backslash e$ into $G$ followed by the contraction of $G$ by $e$ is strong map which is certainly $1-1$ and onto on $\{G\} \backslash\{e\}$.

Conversely, assume $f: G^{\prime} \rightarrow G^{\prime \prime}$ is a $1-1$ strong map from $G^{\prime}$ onto $G^{\prime \prime}$ and assume $r\left(G^{\prime}\right)=r\left(G^{\prime \prime}\right)+1$. Then associated with $f$ there is an onto strong map $\bar{f}$ : $\bar{G}^{\prime} \rightarrow \bar{G}^{\prime \prime}$ between the underlying geometries $\bar{G}^{\prime}$ and $\bar{G}^{\prime \prime}$. This is so since all loops must be mapped into loops so that the 0 element of $\bar{G}^{\prime}$ is sent to the 0 element of $\bar{G}^{\prime \prime}$ and also all points in the same atom in $\bar{G}^{\prime}$ must be mapped to the same lattice element in $\bar{G}^{\prime \prime}$ since inverse images of closed sets are closed. By the Higgs the orem (4.1) we may factor $f$ through $\bar{G}$. The geometry $\bar{G}$ may be viewed as a quotient of $\bar{G}^{\prime} \oplus B_{1}$ where $B_{1}$ is the two element lattice.

At most one atom of $\bar{G}^{\prime}$ is mapped to the 0 element of $\bar{G}^{\prime \prime}$ since rank is decreased by one. There are then two cases to consider:

If no atoms are sent to 0 , then using (5.1) the atoms of $\bar{G}$ are the elements $\left\{\left(a_{i}, 0\right)\right\} \cup(0,1)$ where the $a_{i}$ 's are the atoms of $G^{\prime}$, since the 0 element of $\bar{G}^{\prime}$ is the only preimage of the 0 element of $\bar{G}^{\prime \prime}$. We construct the pregeometry $G$ from $\bar{G}$ on the set of points $\left\{G^{\prime}\right\} \cup\{e\}$ in which the atom $(0,1)$ represents the point $e$ and the atom $\left(a_{i}, 0\right)$ (and 0 element) of $G$ contains the points of the atom $a_{i}$ (and 0 element) of $G^{\prime}$. Then by (5.1), $G^{\prime} \simeq G \backslash e$ and $G^{\prime \prime} \simeq G / e$. Further, $r^{\prime}(G \backslash e)-$ $r^{\prime \prime}(G / e)=1$ so $e$ is not an isthmus or loop of $G$.

If on the other hand an atom $a^{\prime}$ of $\bar{G}^{\prime}$ is sent to 0 , the atoms of $\bar{G}$ are the ele- 
ments $\left(a^{\prime}, 1\right) \cup\left\{\left(a_{i}, 0\right)\right\}$ for all atoms $a_{i} \neq a^{\prime}$ of $G^{\prime}$ since $a^{\prime}$ is the supremum of the two preimages of $0: a^{\prime}$ and 0 , while $a^{\prime}$ is the only atom of $\bar{G}^{\prime}$ which is not minimal for some given image. Construct the pregeometry $G$ from $\bar{G}$ on the point set $\left\{G^{\prime}\right\}$ $\cup\{e\}$ in which for all $a_{i} \neq a^{\prime}$, the atom $\left(a_{i}, 0\right)$ (and 0 element) of $G$ contains the points of the atom $a_{i}$ (and 0 element) of $G^{\prime}$; and in which the atom $\left(a^{\prime}, 1\right)$ contains the points of $a^{\prime} \in G^{\prime}$ as well as the point $e$. Then since $\overline{(0,1)}^{G}=\left(a^{\prime}, 1\right)$ we are done as in the first case.

If $G \backslash e$ and $G / e$ are pregeometries on the same points then using (4.1) the copoints of $G / e$ are exactly those copoints of the constructed $G$ which contain the point $e$ with $e$ then deleted. Also the closed sets of $G \backslash e$ are exactly those sets $A \subseteq\{G\} \backslash\{e\}$ such that $A$ or $A \cup\{e\}$ is closed. Hence the set of copoints, $\underline{D}$, of $G$ is equal to the union $\left(\underline{D}^{\prime} \backslash \underline{D}^{\prime \prime}\right) \cup\left(\underline{D}^{\prime \prime} \times\{e\}\right)$ where $\underline{D}^{\prime \prime}$ is the family of copoints of $G / e$ and $\underline{D}^{\prime}$ is the family of copoints of $G^{\prime} \backslash e$. Hence $G$ is unique up to isomorphism in the above factorization for any 1-1 onto unit rank decreasing strong map $f: G^{\prime} \rightarrow G^{\prime \prime}$.

We mention in passing another consequence of the above theorem.

Corollary 5.3. The partial order $P_{n}$ of isomorphism classes of pregeometries on $n$ points ordered by strong maps ( $G \leq H$ if there exists a strong map $f: G \rightarrow H$ ) is self-dual where $i: \widetilde{P}_{n} \simeq P_{n}$ takes a pregeometry to its (Whitney) dual.

Proof. The covering pairs in $P_{n}$ are the unit rank decreasing strong maps. But $G^{\prime \prime}$ covers $G^{\prime}$ iff there exists a pregeometry $G$ on $n+1$ points such that $G^{\prime} \simeq$ $\underset{\sim}{G} \backslash e$ and $G^{\prime \prime} \simeq G / e$ by $(5.2)$ iff $\widetilde{G^{\prime}} \simeq \widetilde{G} / e$ and $\widetilde{G^{\prime \prime}} \simeq \widetilde{G} \backslash e$ by (4.7) iff $\tilde{G}^{\prime}$ covers $\tilde{G}^{\prime \prime}$.by (5.2).

6. The Tutte polynomial. In $\$ \S 3$ and 4 we noted the importance of the function $t(G)$ taking a pregeometry into the Tutte-Grothendieck ring. In this chapter we will cite some of the properties of this polynomial, called the Tutte polynomial after previous work by Henry Crapo [8].

We note in passing that $t(G)=\Sigma b_{i j} z^{i} x^{j}$ regarded as a generating function has a combinatorial interpretation wherein $b_{i j}$ counts the number of bases with internal activity $i$ and external activity $j$. Relative to a linear ordering $O$ on the points of a pregeometry $G$, a point $p_{1} \in B$ is internally active relative to the bas is $B$ if $p_{1}$ is greater than any other point in the unique bond contained in $(G \backslash B) \cup\left\{p_{1}\right\}$. Dually, $p_{2} \in G \backslash B$ is externally active relative to the basis $B$ (and order $O$ ) if $p_{2}$ is greater than any other point in the unique circuit contained in $B \cup\left\{p_{2}\right\}$. The internal (external) activity of $B$ is then the number of points internally (externally) active relative to $B$ (and $O$ ). This interpretation, dependent as it is on the particular ordering, is explored no further in the present paper.

Theorem 6.1. Assume $t(G)=\sum_{i=0}^{n} \Sigma_{j=0}^{m} b_{i j} z^{i} x^{j}$ is the Tutte polynomial of a pregeometry $G$ where $\Sigma_{j} b_{n j}>0$ and $\Sigma_{i} b_{i m}>0$. Then the following facts bold: 
(a) The rank of $G, r(G)$ is equal to $n$ while $n(G)$, the nullity of $G$ is equal to $m$. Hence $|G|=n+m=\log _{2}\left(\left.t(G)\right|_{z=x=2}\right)$.

(b) $b_{n j}=\delta(j, k)$ iff $G$ bas $k$ loops and $b_{i m}=\delta(i, b)$ iff $G$ bas $b$ isthmuses. If $G^{\prime}$ has $j$ loops then $G^{\prime}=\bigoplus_{k=1}^{j} x \oplus G$ where $G$ bas no loops and $t\left(G^{\prime}\right)=$ $x^{j} t(G)$. Hence no generality is lost if we now assume $G$ to be loopless so $\Sigma_{j} b_{n j}=$ $b_{n 0}=1$.

(c) The number of atoms of $G$ (flats of rank one) is equal to $n+b_{n-1,0}$.

(d) $\Sigma_{j} b_{n-1, j}=m$ while if $G$ bas no istbmuses, $\Sigma_{i} b_{i, m-1}=n$.

Proof. In (a), (b), and (d) the second statement follows from the first and (4.7) which states that $t(\tilde{G})=\Sigma b_{j i} z^{i} x^{j}$.

To prove (a) and (b) we use induction on the number of nonfactors. If $G=$ $\bigoplus_{i=1}^{n} z \oplus \bigoplus_{j=1}^{m} x$ then $t(G)=z^{n} x^{m}$ while $G$ has rank $n$. In addition $b_{n j}=\delta(j, m)$ while $G$ has $m$ loops.

Assuming the result for all pregeometries with $m$ nonfactors, let $G$ have $m+1$ nonfactors one of which is $e$. Then $t(G)=t(G \backslash e)+t(G / e)$. $G$ has rank $n$ with $k$ loops iff $G \backslash e$ has rank $n$ with $k$ loops and $G / e$ has rank $n-1$ iff (by induction) in $G \backslash e$, the coefficient of $z^{n} x^{j}$ is $\delta(j, k)$ while the coefficient for $z^{i} x^{j}$ is 0 for all $i>n$ in $G \backslash e$ and for all $i \geq n$ in $G / e$. The result follows from adding the polynomials $t(G \backslash e)$ and $t(G / e)$. Since $|G|=r(G)+n(G)$ we are done by (4.6) wherein it was proved that $\left.t(G)\right|_{z=x=2}=2|G|$.

We now assume that $G$ is a loopless pregeometry. If $G$ has no nonfactors then $t(G)=z^{n}$ and (c) and (d) hold since $G$ has $n$ atoms while $b_{n-1,0}=\Sigma_{j} b_{n-1, j}=$ $0=m$.

Inductively, let $t(G)=t(G \backslash e)+t(G / e)$. If $e$ is closed in $G$, then $G \backslash e$ has one less atom than $G$ while $G / e$ is loopless so the coefficient of $z^{n-1}$ in $G / e$ is one. If $e^{\prime} \in \bar{e}$ in $G$, then $G \backslash e$ has the same number of atoms as $G$ while $e^{\prime}$ is a loop in $G / e$ so that coefficient of $z^{n-1}$ in $G / e$ is 0 . In any event, by induction $n(G \backslash e)=$ $r(\tilde{G} / e)=m-1=\Sigma_{j} b_{n-1, j}^{\prime}$ in $t(G \backslash e)$, while in $t(G / e), \Sigma_{j} b_{n-1, j}^{\prime \prime}=1$. Both (c) and (d) follow by adding the polynomials $t(G \backslash e)$ and $t(G / e)$.

Lemma 6.2. Let $G$ be a pregeometry of rank $n$. If $f_{n}^{k j}$ counts the number of flats of corank $k$ and nullity $j$ in a rank $n$ pregeometry, and if $e$ is a nonfactor of $G$ then:

$$
f_{n}^{k j}(G)=f_{n}^{k j}(G \backslash e)+f_{n-1}^{k j}(G / e)-\bar{f}_{n-1}^{k, j+1}(G / e)
$$

where $\bar{f}_{n-1}(G / e)$ counts only those closed sets $x$ of G/e which are not closed in $G$.

In particular, if $e$ is a nonfactor of all flats of corank $k$ and nullity $j+1$ in $G$ then $\bar{f}_{n-1}^{k, j+1}(G / e)=f_{n-1}^{k, j+1}(G / e)$. 
Proof. We will use properties of the Tutte decomposition (4.1) and will account for all contributions to the above equation which we denote by $(a)=(b)+$ (c) - (d).

The closed sets of corank $k$ and nullity $j$ in $G$ counted in (a) either contain $e$ and are equicardinal with (c) (since $e$ is not a loop) or do not contain $e$ and are counted in (b). The other flats counted in (b) are those subsets $y$ such that $y \cup e$ is closed in $G$ but $y$ is not. Hence $e$ is not an isthmus of $y \cup e$ and hence $y \cup e$ has corank $k$ and nullity $j+1$ in $G / e$. Such sets $y$ are exactly those sets counted in (d).

Theorem 6.3. Let $G$ be a pregeometry of rank $n$ with Tutte polynomial $t(G)=$ $\Sigma b_{i j} z^{i} x^{j}$. If all $p$ element subsets of $G$ are independent, then the number of flats of $G$ of corank $k$ and nullity $j$ is counted for all $j$ by

$$
f_{n}^{k j}(G)=\sum_{s=k}^{n} \sum_{t=0}^{n-s}(-1)^{t}\left(\begin{array}{c}
n-s \\
t
\end{array}\right)\left(\begin{array}{l}
s \\
k
\end{array}\right) b_{s, j+t}
$$

if $k \geq n-p$ or if $k=0$.

(Note that for $k>n-p$ this gives the obvious: $f_{n}^{k j}(G)=\delta(0, j)\left(\begin{array}{l}n \\ k\end{array}\right)$.)

Proof. For all pre-Boolean algebras, $f_{n}^{k j}\left(B_{n m}\right)=\left(\begin{array}{c}n \\ k\end{array}\right) \delta(j, m)$. But $t\left(B_{n m}\right)=z^{n} x^{m}$, hence $b_{s, j+t}$ is zero unless $s=n$ and $t=m-j$ in which case the formula gives

$$
(-1)^{0}\left(\begin{array}{c}
0 \\
m-\jmath
\end{array}\right)\left(\begin{array}{l}
n \\
k
\end{array}\right)=\left(\begin{array}{l}
n \\
k
\end{array}\right) \delta(j, m)
$$

We will use induction on the number of nonfactors. Since we have proved the theorem for 0 nonfactors above we may assume that the equality holds for all pregeometries with fewer than $q$ nonfactors and consider $G$ with $q$ nonfactors including $e$. We will show that the recursion of (6.2) is valid for all $k$ in the theorem with $\bar{f}$ replaced by $f$. Surely it holds if $k=0$.

By (4.1) a subset $S$ containing a nonfactor $e$ is independent in $G$ iff $S \backslash\{e\}$ is independent in $G / e$ and a subset $S$ disjoint from $e$ is independent in $G$ iff it is independent in $G \backslash e$. Hence all subsets of corank $n-p$ are independent in $G$ iff all subsets of corank $n-p$ are independent in $G \backslash e$ and $G / e$. But all subsets of corank $n-p$ are independent iff all subsets of corank $n-p+1$ are (independent and) closed. If $x$ is a closed set of corank greater than or equal to $n-p+1$, the subgeometry $x$ is a Boolean algebra while if $x$ has corank $n-p$, the subgeometry is the truncation of a Boolean algebra (i.e. free). In any event the recursion of (6.2) for $f_{n}^{k j}$ holds for all $k \geq n-p$ (since in all such subgeometries isthmuses are only of flats of nullity 0 ) and the induction hypothesis may be extended to $G \backslash e$ and $G / e$. Simplifying the dexter we get successively if $t(G \backslash e)=\Sigma b_{i j}^{\prime} z^{i} x^{j}$ and $t(G / e)=\Sigma b_{i j}^{\prime \prime} z^{i} x^{j}$ : 


$$
\begin{aligned}
& f_{n}^{k j}(G \backslash e)+f_{n-1}^{k j}(G / e)-f_{n-1}^{k, j+1}(G / e) \\
& =f_{n}^{k j}(G \backslash e) \\
& \quad+\sum_{s=k}^{n-1} \sum_{t=0}^{n-s}\left[(-1)^{t}\left(\begin{array}{c}
n-s-1 \\
t
\end{array}\right)\left(\begin{array}{l}
s \\
k
\end{array}\right) b_{s, j+t}^{\prime \prime}-(-1)^{t-1}\left(\begin{array}{c}
n-s-1 \\
t-1
\end{array}\right)\left(\begin{array}{l}
s \\
k
\end{array}\right) b_{s, j+t}^{\prime \prime}\right] \\
& =\sum_{s=k}^{n} \sum_{t=0}^{n-s}(-1)^{t}\left(\begin{array}{c}
n-s \\
t
\end{array}\right)\left(\begin{array}{l}
s \\
k
\end{array}\right) b_{s, j+t}^{\prime}+\sum_{s=k}^{n-1} \sum_{t=0}^{n-s}(-1)^{t}\left(\begin{array}{c}
n-s \\
t
\end{array}\right)\left(\begin{array}{l}
s \\
k
\end{array}\right) b_{s, j+t}^{\prime \prime} \\
& =\sum_{s=k}^{n} \sum_{t=0}^{n-s}(-1)^{t}\left(\begin{array}{c}
n-s \\
t
\end{array}\right)\left(\begin{array}{l}
s \\
k
\end{array}\right)\left(b_{s, j+t}^{\prime}+b_{s, j+t}^{\prime \prime}\right)-\left(\begin{array}{l}
n \\
k
\end{array}\right) b_{n j}^{\prime \prime} \\
& =f_{n}^{k j}(G)-0 .
\end{aligned}
$$

Corollary 6.4. Under the bypotheses of the above theorem the number of flats of corank $k$ (the Whitney number of the second kind $w_{n-k}$ ) is given by

$$
w_{n-k}=\left(\begin{array}{l}
n \\
k
\end{array}\right)+\sum_{s=k}^{n-1} \sum_{i=0}^{n-s-1}(-1)^{i}\left(\begin{array}{c}
n-s-1 \\
i
\end{array}\right)\left(\begin{array}{l}
s \\
k
\end{array}\right) b_{s i}
$$

(Again $w_{i}=\left(\begin{array}{c}n \\ i\end{array}\right)$ for all $\left.i<p.\right)$ In particular, if $G$ is a geometry (i.e. $\left.p \geq 2\right)$, $w_{2}=\left(\begin{array}{l}n \\ 2\end{array}\right)+b_{n-2,0}-b_{n-2,1}+(n-1) b_{n-1,0}$.

Proof.

$$
\begin{aligned}
w_{n-k} & =\sum_{s=k}^{n}\left(\begin{array}{l}
s \\
k
\end{array}\right) \sum_{j=0}^{\infty} \sum_{t=0}^{n-s}(-1)^{t}\left(\begin{array}{c}
n-s \\
t
\end{array}\right) b_{s, j+t} \\
& =\sum_{s=k}^{n}\left(\begin{array}{l}
s \\
k
\end{array}\right) \sum_{i=0}^{\infty} \sum_{t=0}^{i}(-1)^{t}\left(\begin{array}{c}
n-s \\
t
\end{array}\right) b_{s i} \\
& =\sum_{s=k}^{n-1}\left(\begin{array}{l}
s \\
k
\end{array}\right) \sum_{i=0}^{\infty}(-1)^{i}\left(\begin{array}{c}
n-s-1 \\
i
\end{array}\right) b_{s i}+\left(\begin{array}{l}
n \\
k
\end{array}\right) \sum_{i=0}^{\infty} b_{n i} .
\end{aligned}
$$

Corollary 6.5. If all $p$ element subsets of $G$ are independent then for all $s \geq n-p$,

$$
b_{s j}=\sum_{\nu=0}^{\infty}\left(\begin{array}{c}
n-1-s+v \\
n-1-s
\end{array}\right) \sum_{q=s}^{\infty}(-1)^{q-s}\left(\begin{array}{l}
q \\
s
\end{array}\right) f^{q, j+v}
$$

where $r(G)=n, f^{k j}$ is the number of flats of $G$ of corank $k$ and nullity $j$, and $b_{s j}$ 
is the coefficient of $z^{s} x^{j}$ in the Tutte polynomial $t(G)$.

In particular, if $m$ is the nullity of $G$, for all $t<p, j>0$,

$$
\begin{aligned}
& b_{n-t, 0}=\left(\begin{array}{c}
m+t-1 \\
t
\end{array}\right) ; \quad b_{n-t, j}=0 \\
& b_{n-p, 0}=\sum_{v=0}^{\infty}\left(\begin{array}{c}
p-1+v \\
p-1
\end{array}\right) f^{n-p, v}+\left(\begin{array}{c}
m+p-1 \\
p
\end{array}\right)-\left(\begin{array}{c}
n+m \\
p
\end{array}\right) ;
\end{aligned}
$$

and

$$
b_{n-p, j}=\sum_{\nu=0}^{\infty}\left(\begin{array}{c}
p-1+v \\
p-1
\end{array}\right) f^{n-p, v+j} .
$$

Proof. Since the formula involves only terms $f^{q, j+v}$ for $q \geq s \geq n-p$, we need only check that the formula inverts the equation for $f_{n}^{k j}$ in (6.3). We will use the symbolic methods of the umbral calculus.

$$
\begin{aligned}
f^{k j}=u^{k} v^{j} & =\sum_{s=k}^{\infty} \sum_{t=0}^{n-s}(-1)^{t}\left(\begin{array}{c}
n-s \\
t
\end{array}\right)\left(\begin{array}{l}
s \\
k
\end{array}\right) x^{s} y^{j+t} \\
& =(1-y)^{n} y^{j} \sum_{s=k}^{\infty}\left(\begin{array}{l}
s \\
k
\end{array}\right)\left(\frac{x}{1-y}\right)^{s} \\
& =(1-y)^{n} y^{j}\left(\frac{x}{1-y}\right)^{k}\left(1-\frac{x}{1-y}\right)^{-k-1} \\
& =\frac{(1-y)^{n+1} x^{k} y^{j}}{(1-y-x)^{k+1}} \quad\left(x^{s} y^{t}=b_{s t}\right) .
\end{aligned}
$$

Similarly,

$$
\begin{aligned}
b_{s j} & =x^{s} y^{j}=\sum_{r=0}^{\infty}\left(\begin{array}{c}
n-1-s+r \\
n-1-s
\end{array}\right) \sum_{q=s}^{\infty}(-1)^{q-s}\left(\begin{array}{l}
q \\
s
\end{array}\right) u^{q} v^{j+r} \\
& =\frac{u^{s} v^{j}}{(1+u)^{s+1}(1-v)^{n-s}} \quad\left(u^{k} v^{j}=f^{k j}\right) .
\end{aligned}
$$

Composing we get:

$$
\begin{aligned}
\sum_{s=k}^{\infty} \sum_{t=0}^{n-s}(-1)^{t} & \left(\begin{array}{c}
n-s \\
t
\end{array}\right)\left(\begin{array}{l}
s \\
k
\end{array}\right) \frac{u^{s} v^{j+t}}{(1+u)^{s+1}(1-v)^{n-s}} \\
& =\sum_{s=k}^{\infty}\left(\begin{array}{l}
s \\
k
\end{array}\right) \frac{u^{s} v^{j}(1-v)^{n-s}}{(1+u)^{s+1}(1-v)^{n-s}} \\
& =\frac{u^{k} v^{j}}{(1+u)^{k+1}}\left(1-\frac{u}{u+1}\right)^{-k-1}=u^{k} v^{j}=f^{k j}
\end{aligned}
$$


Conversely,

$$
\begin{aligned}
& \sum_{r=0}^{\infty}\left(\begin{array}{c}
n-1-s+r \\
n-1-s
\end{array}\right) \sum_{q=s}^{\infty}(-1)^{q-s}\left(\begin{array}{l}
q \\
s
\end{array}\right) \frac{(1-y)^{n+1} x^{q} y^{j+r}}{(1-y-x)^{q+1}} \\
& \quad=y^{j}(1-y)^{n+1}\left(\frac{x}{1-y-x}\right)^{s}\left(1+\frac{x}{1-y-x}\right)^{-s-1}(1-y)^{-n+s}(1-y-x)^{-1} \\
& \quad=x^{s} y^{j}=b_{s j} .
\end{aligned}
$$

Changing variables in the formula for $b_{s j}$ if the nullity of $G$ is $m$,

$$
b_{n-q, j}=\sum_{v=0}^{\infty}\left(\begin{array}{c}
q-1+v \\
q-1
\end{array}\right) \sum_{r=0}^{q}(-1)^{q-r}\left(\begin{array}{l}
n-r \\
q-r
\end{array}\right) f^{n-r, j+v} .
$$

But every subset of $p-1$ or less elements is closed, hence $f^{n-r, j+v}=$ $\left(\begin{array}{c}n+m \\ r\end{array}\right) \delta(j+v, 0)$ for all $r \leq p-1$ and

$$
b_{n-q, j}=\sum_{r=0}^{q}(-1)^{q-r}\left(\begin{array}{c}
n-r \\
q-r
\end{array}\right)\left(\begin{array}{c}
n+m \\
r
\end{array}\right) \delta(j, 0)=\left(\begin{array}{c}
m+q-1 \\
q
\end{array}\right) \delta(j, 0)
$$

for all $q<p$ as can be seen by comparing the coefficient of $x^{q}$ on either side of the identity $(1+x)^{-(n-q+1)}(1+x)^{n+m}=(1+x)^{m+q-1}$. Further

$$
\begin{aligned}
b_{n-p, j} & =\sum_{v=0}^{\infty}\left(\begin{array}{c}
p-1+v \\
p-1
\end{array}\right) f^{n-p, j+v}+\left(\sum_{r=0}^{p-1}(-1)^{p-r}\left(\begin{array}{c}
n-r \\
p-r
\end{array}\right)\left(\begin{array}{c}
n+m \\
r
\end{array}\right)\right) \delta(j, 0) \\
& =\sum_{v=0}^{\infty}\left(\begin{array}{c}
p-1+v \\
p-1
\end{array}\right) f^{n-p, j+v}+\left(\left(\begin{array}{c}
m+p-1 \\
p
\end{array}\right)-\left(\begin{array}{c}
n+m \\
p
\end{array}\right)\right) \delta(j, 0) .
\end{aligned}
$$

Theorem 6.6. If $|G| \geq n$ and the Tutte polynomial $t(G)$ equals $\Sigma_{s, t} b_{s t} z^{s} x^{t}$ then the following identity bolds among the coefficients $b_{s t}$ :

$$
I_{n}(G)=\sum_{s=0}^{n-1} \sum_{t=0}^{n-s-1}(-1)^{t}\left(\begin{array}{c}
n-s-1 \\
t
\end{array}\right) b_{s t}=0
$$

Furthermore, in the vector space $V$ of linear combinations of Tutte coefficients $\left\{b_{s t}\right\}$, the vectors $\left\{I_{1}, \cdots, I_{n}, \cdots\right\}$ form a basis for the subspace of all linear identities which bold for all but a finite number of Tutte polynomials.

Proof. It is sufficient for an inductive proof to show that $I_{n}(G)=0$ for all $|G|=n$ since if $I_{n}(G)=0$ for all $|G|=k \geq n$ then it also holds for all pre-Boolean algebras $B_{i j}, i+j=k+1>n$, since the only coefficients involved in $I_{n}$ are 
those $b_{s t}$ such that $s+t<n$ 。 On the other hand if $|G|=k+1$ has a nonfactor $e$ then $t(G)=t(G \backslash e)+t(G / e)$, and the latter two polynomials satisfy the induction hypothesis so that $I_{n}(G)=I_{n}(G \backslash e)+I_{n}(G / e)=0$.

Now assume $r(G)=n^{\prime}$ and $n(G)=m^{\prime}$ where $n^{\prime}+m^{\prime}=n . I_{n}\left(B_{n}\right)=0$ vacuously so assume $m^{\prime}>0$. The only flat of corank 0 is $G$, hence using the notation of (6.3), $f_{n}^{0, i}=0$ for $i=0,1, \cdots, m^{\prime}-1$, so we have

$$
\begin{aligned}
0 & =\sum_{i=0}^{m^{\prime}-1}(-1)^{i}\left(\begin{array}{c}
m^{\prime}-1 \\
i
\end{array}\right) f_{n}^{0, i}=\sum_{s=0}^{n^{\prime}} \sum_{i=0}^{m^{\prime}-1} \sum_{t=0}^{n^{\prime}-s}(-1)^{i+t}\left(\begin{array}{c}
m^{\prime}-1 \\
i
\end{array}\right)\left(\begin{array}{c}
n^{\prime}-s \\
t
\end{array}\right) b_{s, i+t} \\
& =\sum_{s=0}^{n^{\prime}} \sum_{j=0}^{n-s-1}(-1)^{j} \sum_{t=0}^{j}\left(\begin{array}{c}
m^{\prime}-1 \\
j-t
\end{array}\right)\left(\begin{array}{c}
n^{\prime}-s \\
t
\end{array}\right) b_{s j} \quad(j=i+t) \\
& =\sum_{s=0}^{n^{\prime}} \sum_{j=0}^{n-s-1}(-1)^{j}\left(\begin{array}{c}
n-s-1 \\
j
\end{array}\right) b_{s j} \\
& =I_{n}(G) \text { since } b_{s j}=0 \text { for all } s>n^{\prime} .
\end{aligned}
$$

Certainly the $I_{n}$ are independent in $V$ since $I_{n+1}=\Sigma_{s=0}^{n}(-1)^{n-s_{b}} b_{s, n-s}+$ $\Sigma_{i+j<n} a_{i j} b_{i j}$ is independent of $\left\{I_{j}\right\}_{j \leq n}$. On the other hand if $I_{n+1}^{\prime}=$ $\Sigma_{i+j \leq n} a_{i j}^{\prime} b_{i j}$ is a relation which is 0 almost everywhere then $-a_{s, n-s}^{\prime}=$ $a_{s-1, n-s+1}^{\prime}$ for all $s=1,2, \cdots, n$ since for the infinite class of pregeometries $B_{s-1, n-s} \oplus C$ (C any connected pregeometry), $b_{s, n-s}=b_{s-1, n-s+1}$ and $b_{i j}=0$ for all other $i+j \leq n$.

We now list some useful properties of the series and parallel connection (3.14). The proofs may all be found in [2].

Lemma 6.7. (a) A pointed pregeometry $(F, p)$ is a series connection $F=$ $S(G, H)$ if and only if $F \backslash p=(G \backslash p)+(H \backslash p)$. Dually, $F$ is a parallel connection $P(G, H)$ iff $F / p=G / p+H / p$.

(b) If $H$ is a loop then $S(G, H)=G$. If $H$ is an istbmus then $P(G, H)=G$.

(c) If $G$ and $H$ each bave at least two points then $S(G, H)(P(G, H))$ is connected if and only if $G$ and $H$ both are.

(d) If $\epsilon \in\{G \backslash \backslash\{p\}$, then $S(G, H) / e=S(G / e, H) ; S(G, H) \backslash e=S(G \backslash e, H)$; $P(G, H) / e=P(G / e, H)$; and $P(G, H) \backslash e=P(G \backslash e, H)$.

The following proposition order generalizes Corollary 14.2 of [10].

Proposition 6.8. If $M_{1}$ is a connected minor of a connected pregeometry $G$, then there exists a sequence of connected minors $M_{1}, M_{2}, \cdots, M_{m}=G$ such that $M_{i-1}=M_{i} \backslash e_{i}$ or $M_{i-1}=M_{i} / e_{i}$ for some $e_{i} \in M_{i}$. 
Proof. We proceed by induction on $|G|$. If $|G|=1$, then $M_{1}=G$ and we are done. Assume that the theorem holds for all $\left|G^{\prime}\right|<n$ and let $M_{1}$ be a connected minor of a pregeometry $G,|G|=n$. Then by definition there exists a sequence of minors $M_{1}=M_{1}^{\prime}, \cdots, M_{m}^{\prime}=G$ such that $M_{i-1}^{\prime}=M_{i}^{\prime} \backslash e_{i}$ or $M_{i-1}^{\prime}=M_{i}^{\prime} / e_{i}$. If $M_{m-1}^{\prime}$ is connected we are done by induction since then there would also exist a connected sequence $\left[M_{1}, M_{m-1}^{\prime}\right]$. So assume $M_{m-1}^{\prime}=G \backslash e=M^{(1)} \oplus \cdots \oplus M^{(k)}$ where each $M^{(k)}$ is connected. Then $M_{1}$ is contained in exactly one of the $M^{(i)}$, assume $M_{1}$ is a minor of $M^{(1)}$. By $(6.7(\mathrm{a})),(G, e)$ is a (connected) series connection, $G=$ $S\left(N^{(1)}, \cdots, N^{(k)}\right)$ where each $N^{(i)}$ is connected and $N^{(i)} \backslash e=M^{(i)}$. By the induction hypothesis we may reduce $\left(N^{(2)}, e\right)$ to a loop $e$ by a connected sequence which induces a connected sequence for $G$ by $(6.7(\mathrm{c}))$ and $(6.7(d))$. But by $(6.7(b))$ the sequence is $\left[G, S\left(N^{(1)}, N^{(3)}, \ldots, N^{(k)}\right)\right]$ and again using the induction hypothesis we can extend the sequence to $M_{1}$.

Corollary 6.9. If $M_{1}$ is a minor of a connected pregeometry $G$, then $t\left(M_{1}\right) \leq$ $t(G)$ (i.e. each coefficient $b_{i j}$ of $t\left(M_{1}\right)$ is less than or equal to the corresponding coefficient of $t(G))$.

Proof. Proceeding by induction, if $|G|=1, G=M_{1}$ and $t(G)=t\left(M_{1}\right)$. If the theorem holds for all $\left|G^{\prime}\right|<n$ and $|G|=n$, then, as in the proof of (6.8), let $M_{1}$, $M_{2}, \cdots, M_{m}=G$ be a sequence of minors such that $M_{i-1}=M_{i} / e_{i}$ or $M_{i} \backslash e_{i}$. If $M_{m-1}$ is connected the result follows by induction: if for example $M_{m-1}=M_{m} / e_{m}$ then $t\left(M_{1}\right) \leq t\left(M_{m-1}\right)=t(G)-t\left(M_{m} \backslash e_{m}\right)$ each coefficient of the latter term being positive.

On the other hand, if, for example, $M_{m}=P\left(M^{(1)}, \cdots, M^{(k)}\right)$ and $M_{1}=M_{1}{ }^{(1)} \oplus$ $\ldots \oplus M_{1}{ }^{(j)}$ we may assume each $M^{(i)}$ contains a nonempty direct sum $\left.M_{1}{ }^{\left({ }^{(}\right)}{ }_{1}\right) \oplus$ $\left.\cdots \oplus M_{1}{ }^{(i}{ }_{s}\right)$ since otherwise we could reduce $M^{(i)}$ to the isthmus $e_{m}$ through a sequence of connected geometries (which would hence have no loops or isthmuses) and the result would follow from the induction hypothesis and the observation that if $e \in M_{m}$ were neither an isthmus nor a loop, then $t\left(M_{m} \backslash e\right) \leq t\left(M_{m}\right)$ and $t\left(M_{m} / e\right) \leq$ $t\left(M_{m}\right)$.

Thus each direct sum factor of $M_{1}$ is partitioned by the $M^{(i)} / e_{m}$ in which it is a minor, hence

$$
\begin{aligned}
t\left(M_{1}\right) & =\prod_{i} t\left(M_{1}{ }^{(i)}\right)=\prod_{j} \prod_{M_{1}{ }^{(i)} \prod_{\subseteq M^{(j)} / e_{m}}} t\left(M_{1}{ }^{(i)}\right) \\
& \leq \prod_{j} t\left(M^{(j)} / e_{m}\right)=t\left(G / e_{m}\right) \leq t(G)
\end{aligned}
$$

where the first inequality follows from induction on each factor.

A similar argument holds if $G$ is a series connection.

Proposition 6.10. If in $t(G), b_{k j}=0$ for all $(k, j) \geq(s, t)$ then $f^{k j}$, the number of closed sets of corank $k$ and nullity $j$, equals 0 for all $(k, j) \geq(s, t)$. 
Proof. If $t(G)=z^{n} x^{m}$, then $b_{k j}=0$ only for all $(k, j) \geq(s, m+1)$ or all $(k, j) \geq$ $(n+1, t)$ and there are no closed sets of nullity greater than $m$ or corank greater than $n$.

Proceeding by induction on the number of nonfactors assume $t(G)=t(G \backslash e)+$ $t(G / e)$. If in $t(G), b_{k j}=0$ for all $(k, j) \geq(s, t)$ then in $t(G \backslash e)$ and $t(G / e)$ respectively $b_{k j}^{\prime}=b_{k j}^{\prime \prime}=0$ for all $(k, j) \geq(s, t)$. If there were a closed set $x$ of corank $u$ and nullity $v$ in $G$, there would either be a closed set of corank $u$ and nullity $v$ in $G / e$ (i.e. $x \backslash e$ ) if $e \epsilon x$ or there would be a closed set of corank $u$ and nullity $v$ in $G \backslash e$ (i.e. $x$ ) if $e \notin x$. In either case by induction we are assured that $(u, v) \unrhd(s, t)$.

7. Characterizations and examples. Certain classes of pregeometries may be characterized by properties of the ir Tutte polynomials. Conversely, for certain classes of pregeometries many of the coefficients of the Tutte polynomials can be computed directly from the cardinality of various families of flats.

Proposition 7.1. All the points of a pregeometry $G$ are loops or istbmuses iff it is completely separable iff it has no nonfactors iff its Tutte polynomial, $t(G)$, bas one term.

The following proposition first appeared in [5]. The proof is new.

Proposition 7.2. A pregeometry $G$ is connected if and only if $G$ is a loop or in $t(G), b_{10}>0$.

Proof. If $G$ is separable $\left(G=G_{1} \oplus G_{2}\right)$ then $t(G)=t\left(G_{1}\right) t\left(G_{2}\right)$. The latter two polynomials have no constant term, hence $t(G)$ can have no first degree terms.

Conversely, if $G$ is connected and not a loop, then it has a minor isomorphic to an isthmus, $z$, and $t(G) \geq t(z)$ by (6.9).

Proposition 7.3. A connected pregeometry $G$ of two or more points is a seriesparallel network [2] (i.e. G has no minor isomorpbic to $L_{4}$, the four point line, or $P_{4}$, the lattice of partitions of a four element set) if and only if $b_{10}=1$ in $t(G)$.

Proof. This characterization is proved in [2].

Corollary 7.4. A pregeometry $G$ without loops or isthmuses is a direct sum of $n$ series-parallel networks iff in $t(G), b_{i j}=\left(\begin{array}{c}i+j \\ i\end{array}\right)$ for all (equivalently for some) $i+j=n$ but $b_{s t}=0$ for all $s+t<n$.

Proof. This is a result of (7.2), (7.3) and the fact that $b_{01}=b_{10}$ (4.8).

Proposition 7.5. In a pregeometry $G$, all $p$ element subsets are independent (i.e. $L(G)$ is distributive up to level $p-1)$ if $b_{n-q, j}=0$ for all $q<p, j>0$.

Proof. This follows in one direction from (6.5) and in the other by (6.10).

The preceding proposition together with the explicit calculations in (6.5) give us characterizations of wide classes of pregeometries by their Tutte polynomials. 
Proposition 7.6. A pregeometry $G$ of rank $n$ is loopless iff in $t(G), b_{i j}=0$ for all $(i, j) \geq(n, 1)$. In addition for sucb pregeometries $b_{n, 0}=1, b_{n-1,0}=f^{n-1}-n$; and $b_{n-1, j}=\Sigma_{v=0}^{\infty} f^{n-1, v+j}$ for all $j>0$ where $f^{n-1, v}$ is the number of atoms with $v+1$ points, and $f^{n-1}$ is the number of atoms of $G$.

Proof. A pregeometry is loopless iff all one element subsets are independent. We apply (6.5) and (7.5).

Similar results hold for pregeometries with loops if we first factor them out as in (6.1).

Proposition 7.7. A pregeometry $G$ of rank $n$ is a geometry iff in $t(G), b_{i j}=0$ for all $(i, j) \geq(n-1,1)$. In addition if the nullity of the geometry $G$ equals $m$ then $b_{n, 0}=1, b_{n-1,0}=m, b_{n-2,0}=\Sigma_{v=0}^{\infty}(v+1) f^{n-2, v}+\left(\begin{array}{c}m+1 \\ 2\end{array}\right)-\left(\begin{array}{c}m+n \\ 2\end{array}\right)$, and $b_{n-2, j}=\Sigma_{v=0}^{\infty}(v+1) f^{n-2}, v+j$; where $f^{n-2, v}$ is the number of $(v+2)$-point lines.

Proof. A pregeometry is a geometry iff all two element subsets are independent. We apply (6.5) and (7.5).

Definition 7.8. A covering $\pi$ of a set $S$ is a family of subsets with union $S$. A covering is a partition of type $n$ (or $n$-partition) of $S$ if every element of $\pi$ or block has at least $n$ elements and every $n$-element subset is in a unique block.

The set $S$, the blocks, and all subsets of $S$ with fewer than $n$ elements form the flats of rank $n+1$ pregeometry. In this pregeometry all subsets of cardinality $n$ are independent. These partitions of type $n$ are discussed in [11] and [10] where they are conjectured to predominate in an enumeration of $n$-point geometries.

Partitions of type $n$ are special in our setting, as the ir Tutte polynomials are completely determined from the cardinalities of the blocks.

Proposition 7.9. $G$ is a partition of type $n-1$ iff $r(G)=n$ and $b_{i j}=0$ for all $(i, j) \geq(2,1)$. In addition if $|G|=n+m$ and $a_{t}$ represents the number of blocks of cardinality $t(t=n-1, n, \cdots, n+m)$, then

$$
\begin{aligned}
& b_{i 0}=\left(\begin{array}{c}
m+n-i-1 \\
n-i
\end{array}\right) \text { for all } i \geq 2 ; \\
& b_{10}=\sum_{\nu=0}^{\infty}\left(\begin{array}{c}
n-2+v \\
n-2
\end{array}\right) a_{v+n-1}+\left(\begin{array}{c}
m+n-2 \\
n-1
\end{array}\right)-\left(\begin{array}{c}
m+n \\
n-1
\end{array}\right) ;
\end{aligned}
$$

and for all $j>0$,

$$
b_{1 j}=\sum_{\nu=0}^{\infty}\left(\begin{array}{c}
n-2+v \\
n-2
\end{array}\right) a_{v+j+n-1}
$$

and

$$
b_{0 j}=\left(\begin{array}{c}
n+m-j-1 \\
n-1
\end{array}\right)-\sum_{v=0}^{\infty}\left(\begin{array}{c}
n-1+v \\
n-1
\end{array}\right) a_{v+j+n-1} .
$$


Proof. The first statement follows from (7.5). Assume $G$ is a partition of type $n-1$. Then the formula in (6.3) for $f_{n}^{k j}$ is valid not only for $k \geq 1$ (since every $(n-1)$-element subset is independent) but also the formula is valid for $k=0$.

Hence (6.3) holds for all $(k, j)$ and may be inverted so that the formula for $b_{s j}$ in $(6.5)$ is valid for all $(s, j)$. Substituting $i$ for $n-q$ in $(6.5), b_{i j}=\left(\begin{array}{c}m+n-i-1 \\ n-i\end{array}\right) \delta(0, j)$ for all $i \geq 2$. Substituting $n-1$ for $p$ and $a_{v+j+n-1}$ for $f^{1, v+j}$ the formulas for $b_{1 j}$ follow from those in (6.5). Finally, for all $j>0$,

$$
\begin{aligned}
b_{0 j} & =\sum_{q=0}^{\infty}(-1)^{q} \sum_{v=0}^{\infty}\left(\begin{array}{c}
n-1+v \\
n-1
\end{array}\right) f^{q, j+v} \\
& =\sum_{\nu=0}^{\infty}\left(\begin{array}{c}
n-1+v \\
n-1
\end{array}\right) f^{0, j+v}-\sum_{\nu=0}^{\infty}\left(\begin{array}{c}
n-1+v \\
n-1
\end{array}\right) f^{1, j+v}+\sum_{j>0, i \geq 2} c_{i j} f^{i j} .
\end{aligned}
$$

But $f^{0, j+v}=\delta(j+v, m)$ hence the first sum equals $\left(\begin{array}{c}n+m-j-1 \\ n-1\end{array}\right) ; f^{1, j+v}=a_{v+j+n-1}$ in the second sum; and each $f^{k j}$ in the third sum equals zero.

We may apply (7.9) to some important special cases: partitions, planar geometries, and free geometries.

The isomorphism classes of loopless rank two pregeometries are in 1-1 correspondence with integer partitions $p=p_{1}+\cdots+p_{k}, p_{1} \geq p_{2} \geq \cdots \geq p_{k}>0$ where $p_{i}$ corresponds to the cardinality of an atom and where $k \geq 2$. To every such partition there corresponds a unique Ferrers dual partition, $p=p_{1}^{\prime}+\cdots+p_{p_{1}}^{\prime}$ where $p_{i}^{\prime}$ is the number of integers $p_{j}$ greater than or equal to $i$.

Proposition 7.10. If $t(G)=b_{i j} z^{i} x^{j}$ is the Tutte palynomial of the pregeometry corresponding to the integer partition $p=p_{1}+p_{2}+\cdots+p_{k}$ with Ferrers dual $p=$ $p_{1}^{\prime}+\cdots+p_{p_{1}}^{\prime}$ then $b_{2 j}=\delta(0, j) ; b_{10}=p_{1}^{\prime}-2 ; b_{1 j}=p_{j}^{\prime}$ and $b_{0 j}=p-j-1-$ $\Sigma_{u \geq 1} u a_{u+j}=p-j-1-\Sigma_{i>j} p_{i}^{\prime}$.

Proposition 7.11. A planar (rank 3) geometry $G$ of nullity $m$ with $a_{i}$ lines of cardinality $i$ is characterized by its Tutte polynomial: $b_{3 j}=\delta(0, j) ; b_{2 j}=m \delta(0, j)$; $b_{10}=\Sigma_{v=0}^{\infty}(v+1) a_{v+2}+\left(\begin{array}{c}m+1 \\ 2\end{array}\right)-\left(\begin{array}{c}m+n \\ 2\end{array}\right) ;$ and for all $j>0$,

$$
b_{1 j}=\sum_{\nu=0}^{\infty}(v+1) a_{v+j+2} ; \quad b_{0 j}=\left(\begin{array}{c}
m-j+2 \\
2
\end{array}\right)-\sum_{v=0}^{\infty}\left(\begin{array}{c}
v+2 \\
2
\end{array}\right) a_{v+j+2} .
$$

Using (6.4) or summing the $a_{i}$ 's one sees that there are $n+b_{20}$ points and $2 b_{20}+b_{10}-b_{11}+n$ lines. Hence, subtracting we get $b_{20}+b_{10}-b_{11}=2 b_{01}-$ $b_{02} \geq 0$ and is equal to 0 if and only if $G$ is a projective plane (modular).

Proposition 7.12. For $n>1$ let $F^{n m}$ represent the partition of type $n-1$ of cardinality $n+m$ in which every $(n-1)$-element subset is a block. We call $F^{n m}$ the free geometry of rank $n$ and nullity $m$. Note that $F^{n m}$ represents the mth truncation of the Boolean algebra $B_{n+m}$. Free geometries are characterized as those for which $b_{i j}=0$ for all $i j \neq 0$ in $t(G)$, and in particular 


$$
t\left(F^{n m}\right)=\sum_{p=0}^{n-1}\left(\begin{array}{c}
m-1+p \\
m-1
\end{array}\right) z^{n-p}+\sum_{q=0}^{m-1}\left(\begin{array}{c}
n-1+q \\
n-1
\end{array}\right) x^{m-q} .
$$

Proposition 7.13. Let $f$ be any Tutte (group) invariant such that $f$ is greater when evaluated on a free (pre)geometry of rank $n$ and nullity $m$ than on the preBoolean algebra of the same rank and nullity for all $n m>0$ (i.e. $f\left(F^{n m}\right)>f\left(B_{n m}\right)$ ). Then $f\left(F^{n m}\right)>f(G)$ for all other pregeometries $G$ of rank $n$ and nullity $m$. (Similar results bold for any other relation compatible with addition.)

Proof. If $G$ is not a pre-Boolean algebra it has a nonfactor $e$ and $f(G)=$ $f(G \backslash e)+f(G / e)$. But $F^{n m}$ decomposes into $F^{n, m-1}$ and $F^{n-1, m}$ (and by (7.12) it is the only pregeometry which does so). Hence by induction on cardinality, $f\left(F^{n, m-1}\right) \geq f(G \backslash e)$ and $f\left(F^{n-1, m}\right) \geq f(G / e)$ with at least one of the inequalities strict.

For given rank and cardinality the number of bases, spanning sets and independent sets are clearly maximized by free geometries; $|\mu|$ and $\beta$ are also.

Corollary 7.14. For all pregeometries of rank $n>1$ and cardinality $n+m$,

$$
|\mu(G)| \leq\left(\begin{array}{c}
m+n-1 \\
n-1
\end{array}\right) \text { and } \beta(G) \leq\left(\begin{array}{c}
m+n-2 \\
n-1
\end{array}\right) .
$$

Further, equality bolds if and only if $G=F^{n m}$.

Proof. Evaluating the Tutte polynomial of (7.12), if $m>0, \beta\left(F^{n m}\right)=b_{10}=$ $\left(\begin{array}{c}n+m-2 \\ n-1\end{array}\right)>0$; and $\left|\mu\left(F^{n m}\right)\right|=\left.t\left(F^{n m}\right)\right|_{z=1, x=0}=\sum_{p=0}^{n-1}\left(\begin{array}{c}m-1+p \\ p\end{array}\right)=\left(\begin{array}{c}m+n-1 \\ n-1\end{array}\right)>1$. We may now apply (7.13).

We conjecture that the Tutte polynomial $t(G)$ of a connected pregeometry is prime in the unique factorization domain $Z[z, x]$ although the characteristic polynomial is not. This would imply that $F$ or another pregeometry with the same Tutte polynomial is a direct sum factor of $G$ iff $t(F)$ divides $t(G)$.

A small partial result in this direction is that if $G$ is a series-parallel network (or any pregeometry $G$ such that $\beta(G)$ is odd) it could not be factored into positive polynomials since $\left.t(G)\right|_{z=2, x=2}=2^{n+m}$ by (4.6) so that any nonconstant factor when evaluated at $z=x=2$ would be a power of 2 and hence would have to have an even constant term.

8. Two counterexamples. We may now ask just how strong an invariant of a pregeometry $G$ the Tutte polynomial $t(G)$ is. Its strength is attested to by the fact that if $G$ is a rank two pregeometry and $t(G)=t(H)$ then $G \simeq H$. (This follows from factoring out loops as in (6.1) and deriving the dual partition numbers $p_{j}^{\prime}$ from the coefficients $b_{1 j}(7.10)$.) Tutte equivalence, however, is weaker than isomorphism. (Hence converses to propositions like (6.9) are generally false.)

Proposition 8.1. Let $G$ be the rank three geometry with points $a_{1}, a_{2}, \cdots, a_{6}$ 
and two nontrivial copoints $\left\{a_{1}, a_{2}, a_{3}\right\}$ and $\left\{a_{4}, a_{5}, a_{6}\right\}$. Let $H$ be the rank three geometry on the same set of points with the nontrivial copoints $\left\{b_{1}, b_{2}, b_{3}\right\},\left\{b_{1}\right.$, $\left.b_{4}, b_{5}\right\}$. Then $G \neq H$ but $t(G)=t(H)$.

No smaller pregeometries $G^{\prime}, H^{\prime}$ bave this same property. Neither do any other geometries of the same cardinality.

Proof. Surely $G \neq H$ since every point of $G$ is on exactly one three point line while in $H, b_{1}$ is on two three point lines (and $b_{6}$ is on none). We will prove that $t(G)=t(H)$ using three methods by way of illustrating some of the previous material.

(i) $G \backslash a_{4}$ and $H \backslash a_{4}$ are isomorphic, each being the planar five point geometry with one three point line. Also $G / a_{4}$ and $H / a_{4}$ are isomorphic, each corresponding to the integer partition $2+1+1+1=5$. Hence $t(G)=t\left(G \backslash a_{4}\right)+t\left(G / a_{4}\right)=$ $t\left(H \backslash a_{4}\right)+t\left(H / a_{4}\right)=t(H)$.

(ii) $G$ and $H$ are both geometries on six points with exactly two three point lines and nine two point lines. But by (7.11) all the coefficients $b_{i j}$ in the Tutte polynomial of a planar geometry are derivable from the ranks and cardinalities of the various flats.

(iii) Embedded in the real plane,

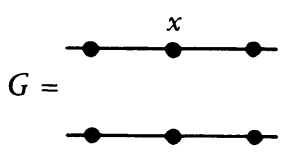

and

$$
H=\left\{\begin{array}{c}
x \\
\bullet
\end{array}\right.
$$

Deleting and contracting the point $x$ gives the desired result.

Since $t(G)=t(H)$ iff $t(\tilde{G})=t(\widetilde{H})$ by (4.7) one sees that no pair of smaller nonisomorphic pregeometries have the same Tutte polynomial since if $|G| \leq 5$, either $G$ or $\tilde{G}$ has rank less than or equal to two and by the remarks preceding this proposition, $G$ is characterized up to isomorphism by its Tutte polynomial.

It is also easy to check that among the planar six point geometries only $G$ and $H$ have the same number of nontrivial lines of the same cardinality.

It is instructive to show in light of (5.2) how the composite strong map $f_{G}$ : $G \backslash a_{4} \rightarrow G / a_{4}$ resulting from the decomposition $G<G \backslash a_{4}+G / a_{4}$ differs from the corresponding strong map $f_{H}$. Pictorially,

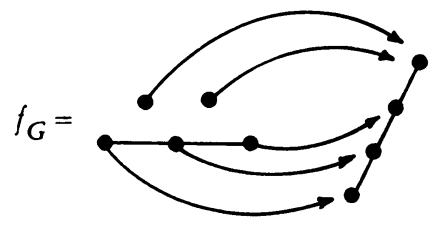

and

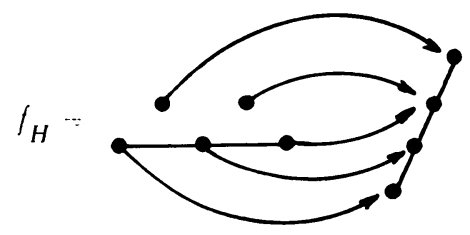


so the two maps differ since in $f_{G}$ the preimage of the two point atom is disjoint from the three point line and in $f_{H}$ it is not.

Since series-parallel networks may be characterized by the Tutte polynomial, it becomes a relevant question to ask if other important classes of geometries (binary, graphic, unimodular, planar graphic, transversal, etc.) may be so characterized. One may also ask if from the Tutte polynomial one can enumerate the number of flats, $f^{k j}$, indexed by corank and nullity as one can for $n$-partitions (7.9). All these hopes are dampened by the following example.

Proposition 8.2. Let $J$ be the rank three pregeometry with points $a_{1}, a_{2}, \cdots$, $a_{7}$ and nontrivial lines $\left\{a_{1}, a_{2}, a_{3}, a_{4}\right\},\left\{a_{1}, a_{5}, a_{6}, a_{7}\right\},\left\{a_{2}, a_{6}, a_{7}\right\},\left\{a_{3}, a_{6}, a_{7}\right\}$, and $\left\{a_{4}, a_{6}, a_{7}\right\}$.

Let $P_{4}^{\prime}$ be the pregeometry with one atom of two points whose underlying geometry is the geometry of partitions of a four element set.

Then $t(J)=t\left(P_{4}^{\prime}\right)$, the Tutte polynomial calculated in (3.4). But $J$ is not binary (and bence not unimodular, graphic, or planar) but $P_{4}^{\prime}$ is all of these. Also $J$ is transversal while $P_{4}^{\prime}$ is not even the contraction of a transversal pregeometry. In addition $P_{4}^{\prime}$ and $J$ bave different numbers of circuits, two point lines, lines, and flats. Such differences also appear in $\tilde{J}$ and $\tilde{P}_{4}^{\prime}$ both of which are geometries.

Proof. In an embedding in the real plane:
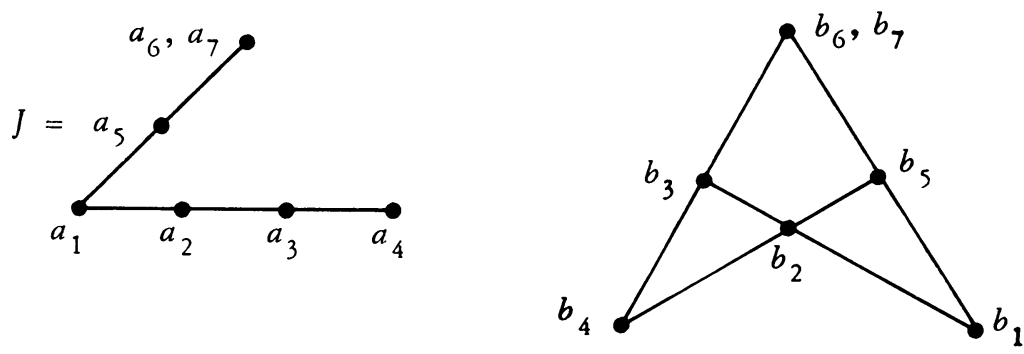

The deletions $J \backslash a_{4}$ and $P_{4}^{\prime} \backslash b_{4}$ are isomorphic by the strong map $f\left(a_{i}\right)=b_{i}, i \neq 4$. In addition $J / a_{4}$ and $P_{4} / b_{4}$ both represent the integer partition $3+2+1=6$ and hence are isomorphic. Therefore, $t(J)=t\left(J \backslash a_{4}\right)+t\left(J / a_{4}\right)=t\left(P_{4}^{\prime} \backslash a_{4}\right)+t\left(P_{4}^{\prime} / a_{4}\right)=$ $t\left(P_{4}^{\prime}\right)$.

The subset $\left\{a_{1}, a_{2}, a_{3}, a_{4}\right\}$ of $J$ is a four atom line and hence by the representation the orem for binary lattices [10], $J$ is not binary (and hence not graphic). On the other hand, we shall see later (11.1) that $P_{4}^{\prime}$ is the geometry of contractions of the planar graph $K_{4}^{\prime}$ :

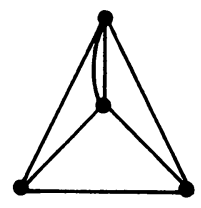


and so $P_{4}^{\prime}$ is planar graphic and hence unimodular and binary. It is well-known that $P_{4}$ (and hence $P_{4}^{\prime}$ ) is not transversal, while $\left\{a_{1}, a_{2}, a_{3}, a_{5}\right\},\left\{a_{2}, a_{3}, a_{4}\right\}$ and $\left\{a_{5}, a_{6}, a_{7}\right\}$ present $J$ as a transversal pregeometry.

Although $\mathrm{J}$ and $\mathrm{P}_{4}^{\prime}$ have the same number of atoms (6), four point lines (2), and three point lines ( 3 ); $J$ has the three two point lines $\left\{a_{2}, a_{5}\right\},\left\{a_{3}, a_{5}\right\}$, and $\left\{a_{4}, a_{5}\right\}$ while $\left\{b_{4}, b_{1}\right\}$ and $\left\{b_{3}, b_{5}\right\}$ are the only two point lines of $P_{4}^{\prime}$. Also, $P_{4}^{\prime}$ has 5 four point circuits while $J$ has six.

Since neither $P_{4}^{\prime}$ nor $J$ have any copoint of five or more points, $\widetilde{P}_{4}^{\prime}$ and $\tilde{J}$ are geometries which are Tutte equivalent but have differences in circuits, copoints, and representability.

We remark that an interesting problem would be to find other conditions on pregeometries which would guarantee that Tutte equivalence would be as strong as isomorphism. For instance it is not known whether two inequivalent graphic (11) (or even binary) geometries can have the same Tutte polynomial (they can have the same characteristic polynomial). Such investigation should lend insight into such areas as the Ulam graph reconstruction conjecture.

In the next four sections we explore the induced Tutte-Grothendieck ring for some important hereditary classes of pregeometries-chain groups over finite fields (especially $G F(2)$, the two element field), graphic pregeometries, and orientable (unimodular) pregeometries. (An hereditary class of pregeometries is one closed under arbitrary minors, i.e. if $G$ is in the class and $p \in G$ then $G \backslash p$ and $G / p$ are also in the class.)

For these classes the subbidecomposition inherited from $\underline{T}$ can be looked at in a new perspective and classic theorems concerning certain invariants can be furnished with new proofs and sometimes generalized by use of the inherited decomposition structure of $\underline{T}\left(S^{\prime}\right)$ (i.e. the Tutte decomposition restricted to one of above classes).

9. Chain groups and the critical problem.

Definition 9.1. Let $V_{n}$ be a vector space of dimension $n$ over a fixed finite field $G F(q)$. For a spanning set of vectors $S \subseteq V_{n}$ not including the zero vector (i.e. $S$ is contained in no proper subspace and $0 \notin S$ ) we define $G(S)$, the geometry spanned by $S$. The closed sets of $G(S)$ are the subspaces of $V_{n}$ spanned by subsets of $S$ (those subspaces which have a basis in $S$ ). Such geometries are called chain groups.

If $s, s^{\prime} \in S$ are both in the same one-dimensional subspace or atom (i.e. $s^{\prime}=$ as, $\alpha \in q \backslash\{0\})$, then $G(S)=G(S \backslash\{s\})$ since any basis $B$ for a subspace containing $s$ may be replaced by $(B \backslash\{s\}) \cup\left\{s^{\prime}\right\}$. Hence $G(S)=G\left(S^{\prime}\right)$ where $S^{\prime} \subseteq S$ has no two dependent vectors. If $S=V_{n}^{\prime}=V_{n} \backslash\{0\}$ (or $\lambda \cap S \neq \varnothing$ for all atoms $\lambda \in V_{n}$ ) then $L(G(S))$ is the modular lattice of the projective space associated with $V_{n}$. Thus 
any chain group $G(S)$ can be thought of as a subgeometry (sequence of deletions of ' dimensional subspaces) of $G\left(V_{n}^{\prime}\right)$ and we will henceforth consider it as such.

Proposition 9.2. Let $S$ be a spanning subset of lines of $V_{n}$, and let $G(S)$ be the associated geometry. Then if $\lambda \in S$ is not an istbmus of $G(S)$, then $G(S \backslash \lambda)=$ $G(S) \backslash \lambda$ and $G(S / \lambda)=G(S) / \lambda$ where $S / \lambda$ is the projection of $\{S\} \backslash\{\lambda\}$ from the atom (one dimensional subspace) $\lambda . S_{1}$ and $S_{2}$ lie in complementary subspaces of $V_{n}$ (i.e. $S_{1} \subseteq V^{\prime}, S_{2} \subseteq V^{\prime \prime}$, and $\left.V=V^{\prime} \oplus V^{\prime \prime}\right)$ if and only if $G\left(S_{1} \cup S_{2}\right)=G\left(S_{1}\right) \oplus G\left(S_{2}\right)$.

Proof. By definition $G(S)$ is the subgeometry $G\left(V_{n}\right) \backslash\left(\left\{V_{n}\right\} \backslash S\right)$, hence

$$
G(S) \backslash \lambda=\left(G\left(V_{n}\right) \backslash\left(\left\{V_{n}\right\} \backslash S\right)\right) \backslash \lambda=G\left(V_{n}\right) \backslash\left(\left\{V_{n}\right\} \backslash(S \backslash \lambda)\right)=G(S \backslash \lambda) .
$$

The flats of rank $k$ in the contraction $G(S) / \lambda$ are the flats of rank $k+1$ in the vector space $V_{n}$ which have a bas is in $S$ and contain the atom $\lambda$. But this is the geometry $G(S / \lambda)$ whose closed sets are subspaces of $V_{n} / \lambda \simeq V_{n-1}$ spanned by atoms of $V_{n} / \lambda$ which are projections of two dimensional subspaces in $V_{n}$ containing $\lambda$ and another atom of $S$.

If $S_{1} \subseteq V^{\prime}, S_{2} \subseteq \dot{V}^{\prime \prime}, V=V^{\prime} \oplus V^{\prime \prime}$, and $S_{1} \cup S_{2}$ spans $V$ then for every pair of bases $B_{1} \subseteq G\left(S_{1}\right)$ and $B_{2} \subseteq G\left(S_{2}\right), B_{1} \cup B_{2}$ is independent and spans $V$, hence is a basis for $G\left(S_{1} \cup S_{2}\right)$. Hence $G\left(S_{1} \cup S_{2}\right)=G\left(S_{1}\right) \oplus G\left(S_{2}\right)$. Conversely if $G\left(S_{1} \cup S_{2}\right)=G\left(S_{1}\right) \oplus G\left(S_{2}\right)$ then let $V^{\prime}=\bar{S}_{1}$ and $V^{\prime \prime}=\bar{S}_{2}$ in $V_{n}$. Then $V=V^{\prime}+V^{\prime \prime}$ since $S_{1} \cup S_{2}$ spans $V_{n}$. But $r(V)=r\left(S_{1} \cup S_{2}\right)=r\left(S_{1}\right)+r\left(S_{2}\right)=r\left(V^{\prime}\right)+r\left(V^{\prime \prime}\right)$ so $V=V^{\prime} \oplus V^{\prime \prime}$.

Definition 9.3. The critical problem as formulated in [10] for a set of vectors $S$ in a vector space $V_{n}(0 \notin S)$ is the extraction of the critical exponent, $c$-the minimal number of projective hyperplanes $H_{1}, \cdots, H_{c}$ such that $H_{1} \cap \ldots \cap H_{c} \cap$ $S=\emptyset$. Since every nonzero linear functional has a hyperplane for its kernel and every hyperplane is the kernel for some linear functional, the critical problem is equivalent to finding the minimal number $c$ of linear functionals, $f_{1}, \cdots, f_{c}$ which distinguish the set $S$, i.e. which for all $s \in S$ there is a functional $f_{i}(0 \leq i \leq c)$ such that $f_{i}(s) \neq 0$.

Lemma 9.4. Let $S$ be a spanning set of vectors for a vector space $V$ and let $N(S, k)$ be the set of ordered $k$-tuples of linear functionals of $V$ which distinguish the set $S$. Then $(-1)^{r(V)}|N(S, k)|$ is a Tutte bidecomposition invariant on the geometry $G(S)$.

Proof. For any two dependent vectors $s, s^{\prime} \in S$ and any linear functional $f$; $f(s)=0$ iff $f\left(s^{\prime}\right)=0$. Hence $\left(f_{1}, \cdots, f_{k}\right)$ distinguishes $S$ iff it distinguishes $S \backslash s$. But by the remarks following $(9.1), G(S)=G(S \backslash s)$, so we may delete any point of $S$ which is in an atom with another point of $S$ and change ne ither $G(S)$ nor $N(S, k)$. We may thus assume that at most one $s \in S$ lies in any atom of $V_{n}$. 
If $\lambda \in S$ is not an isthmus of $G(S)$, we may partition $N(S \backslash \lambda, k)$ into two setsthose which vanish on $\lambda$ and those $k$-tuples that contain an $f_{i}$ such that $f_{i}(\lambda) \neq 0$. The latter set is exactly those $k$-tuples which distinguish $\lambda$ as well as $S \backslash \lambda$ and hence form $N(S, k)$. The former set is equicardinal with $N(S / \lambda, k)$ as is shown below.

Extend $\lambda$ to a basis $B$ for $V_{n}$. We then have the dual basis $B^{*}$. which includes the functional $f_{\lambda}$. It is readily seen that $B^{*} \backslash\left\{f_{\lambda}\right\}$ can be viewed as a basis for $V_{n} / q \lambda$ (where $q \lambda$ is the atom of $V_{n}$ spanned by $\lambda$ ) as well as a basis for those functionals on $V_{n}$ which vanish on $\lambda$. A $k$-tuple of such functionals distinguishes the points $S \backslash\{\lambda\}$ in $V_{n}$ iff in $G\left(V_{n}^{\prime}\right)$ their respective hyperplanes intersect $S$ in $\lambda$ iff the corresponding hyperplanes in $V_{n} / q \lambda$ distinguish $S / \lambda$.

Since $S$ and $S \backslash \lambda$ span the $n$-dimensional space $V_{n}$ and $S / \lambda$ spans an $(n-1)$ dimensional space we see that the identity $(-1)^{n}|N(S, k)|=(-1)^{n}|N(S \backslash \lambda, k)|+$ $(-1)^{n-1}|N(S / \lambda, k)|$ as shown above proves the lemma.

If $V=V^{\prime} \oplus V^{\prime \prime}, S_{1} \subseteq V^{\prime}$, and $S_{2} \subseteq V^{\prime \prime}$, then picking a basis for $V$ (and hence for the dual space $V^{*}$ ) from $V^{\prime} \cup V^{\prime \prime}$, and functional $f$ on $V$ is equal to $f^{\prime}+f^{\prime \prime}$ where $f^{(i)}$ is a functional on $V^{(i)}$ and $\left(f^{\prime}+f^{\prime \prime}\right)(s)=f^{(i)}(s)$ if $s \in S^{(i)}, i=1,2$.

Such a functional $f$ distinguishes $S_{1} \cup S_{2}$ iff $f^{\prime}$ distinguishes $S_{1}$ and $f^{\prime \prime}$ distinguishes $S_{2}$. Hence

$$
(-1)^{r(V)}|N(S, k)|=(-1)^{r\left(V^{\prime}\right)}\left|N\left(S_{1}, k\right)\right|(-1)^{r\left(V^{\prime \prime}\right)}\left|N\left(S_{2}, k\right)\right| .
$$

Theorem 9.5. If $S$ spans $V$, then the number of k-tuples of functionals on $V$ which distinguish $S,|N(S, k)|$, equals $\chi\left(S, q^{k}\right)$ where $\chi(S, \lambda)$ is the characteristic polynomial of $G(S)$ (4.2). Hence if $k=c$, then the number of subsets of byper-

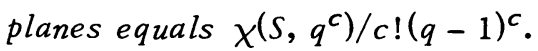

Proof. By the previous lemma $(-1)^{r(V)}|N(S, k)|$ is a Tutte bidecomposition invariant. If $G(S)$ is a loop $x$, it is the origin of a 0 -dimensional vector space and no functional can distinguish it. Hence $(-1)^{0}|N(x, k)|=0$. If $G(S)$ is an isthmus $z$, all $q^{k} k$-tuples of linear functionals distinguish it except the $k$-tuple of all 0 functionals, hence $(-1)^{1}|N(z, k)|=1-q^{k}$. By the fundamental TutteGrothendieck theorem (3.6),

$$
(-1)^{r(V)}|N(S, k)|=\left.t(G)\right|_{z=1-q^{k}, x=0}=\left.(-1)^{r(V)} \chi(S, \lambda)\right|_{\lambda=q^{k}} .
$$

When $k$ is the critical exponent $c$, the $c$ functionals correspond to distinct hyperplanes.

10. Binary pregeometries. A new characterization of binary pregeometries (those which can be coordinatized over the field $G F(2)$ ) results in a theorem generalizing the two-color theorem for planar graphs in its full strength to binary pregeometries. 
Proposition 10.1 Let $G$ be a binary pregeometry and let $C=\left\{c_{1}, \cdots, c_{n}\right\}$ be a circuit of $G$. Then for all points $e \in G \backslash C, C$ is either a circuit or a direct sum of two circuits $C_{1}$ and $C_{2}$ in the contraction $G / e$. In the latter case both $C_{1} \cup$ $\{e\}$ and $C_{2} \cup\{e\}$ were circuits of $G$.

Proof. Let $G=C \cup\{e\}$ where $C$ is a circuit. If $e$ is an isthmus or loop of $G$ then $G / e=C$ and the proposition holds. Otherwise $G$ has nullity two and its dual $\widetilde{G}$ is a binary pregeometry of rank two. In $\widetilde{G}, C$ is a bond which makes $e$ a copoint (atom). A binary pregeometry has been characterized [10] as one in which every coline contains $\underset{\sim}{\sim}$ most three copoints. Hence $\tilde{G}$ has two other atoms $C_{1}$ and $\underset{\sim}{C_{2}}$ which partition $\tilde{G} \backslash e=C$ (since $e$ is not a loop of $G$ hence not an isthmus of $\tilde{G}$ ). Therefore in the pregeometry $\tilde{G} \backslash e$, both are bonds while in $G, C_{1} \cup\{e\}$ and $C_{2} \cup$ $\{e\}$ were both bonds. But this means that in $\widetilde{G} \backslash e=G / e, C$ is partitioned as the direct sum of the two circuits $C_{1}$ and $C_{2}$ and both $C_{1} \cup\{e\}$ and $C_{2} \cup\{e\}$ were circuits of $G$.

For a more general $G$, if $C$ is a circuit of $G$ and $e \in\{G\} \backslash C$, then $(G / e) \backslash(\{G\} \backslash(C \cup\{e\}))=(G \backslash(\{G\} \backslash(C \cup\{e\}))) / e$ since both are defined by the derived closure operator $J(A)=\overline{A \cup e} \backslash(\{G\} \backslash C)$ for all $A \subseteq C$ where $\overline{A \cup e}$ is the closure in $G . C$ is a circuit of $G$ iff it is a circuit of any subgeometry of $G$ containing it (a circuit is defined wholly internally in terms of rank and cardinality of its subsets) hence we may delete everything except $C$ and $e$ before we contract $e$ and the above special case applies.

Note that if $L_{4}$ is the four point line and $e \in L_{4}$, then $L_{4} \backslash e$ is a circuit but in $L_{4} / e$ all three two point subsets are circuits. Hence $G$ is binary iff $(10.1)$ holds for all minors of $G$.

Lemma 10.2. Let $G$ be a binary pregeometry. Then

$$
f(G)= \begin{cases}(-1)^{r(G)} & \text { if } G \text { bas all even circuits, } \\ 0 & \text { if } G \text { bas an odd circuit, }\end{cases}
$$

is a Tutte invariant.

Proof. If $G=G_{1} \oplus G_{2}$ then the circuit set of $G, \underline{C}(G)$ is the disjoint union of $\underline{C}\left(G_{1}\right)$ and $\underline{C}\left(G_{2}\right)$. Hence $\underline{C}(G)$ contains all even circuits iff $\underline{C}\left(G_{1}\right)$ and $\underline{C}\left(G_{2}\right)$ both do iff $f\left(G_{1}\right)$ and $f\left(G_{2}\right)$ are both nonzero in which case $(-1)^{r\left(G_{1}\right)}(-1)^{r\left(G_{2}\right)}=$ $(-1)^{r\left(G_{1} \oplus G_{2}\right)}=(-1)^{r(G)}$. Otherwise, $f\left(G_{1}\right)=0$ or $f\left(G_{2}\right)=0$ and $f(G)=0=f\left(G_{1}\right) f\left(G_{2}\right)$.

We must prove that $f(G)=f(G \backslash e)+f(G / e)$ for any nonfactor $e \in G$. Since $r(G)=r(G \backslash e)=r(G / e)+1$, dividing the equation through by $(-1)^{r(G)}$ we get

$$
|f(G)|=|f(G \backslash e)|-|f(G / e)| \text {. }
$$

Assume $|f(G)|=1$. Then $G$ has all even circuits and so does $G \backslash e$ (since for all $C \subseteq G \backslash e, C$ is a circuit of $G$ if and only if it is a circuit of $G \backslash e$ ). Since $e$ is 
not an isthmus, $e$ is in some (even) circuit of $G$ and so $C / e$ is a circuit (of odd length) in $G / e$ and $(* *)$ becomes $1=1-0$.

If $f(G)=0$ and $f(G \backslash e)=0$ then $G$ must contain an odd circuit $C$ disjoint from $e$. By the above proposition (10.2) the set $C$ is either a circuit in $G / e$ or the direct sum of two circuits $C_{1}$ and $C_{2}$ in which case $\left|C_{1}\right|+\left|C_{2}\right|=|C|$. Since $C$ is odd one of the summands and hence one of the circuits in $G / e$ is odd and (**) is evaluated as $0=0-0$.

Finally let $f(G)=0$ and $|f(G \backslash e)|=1$. Then all circuits not containing $e$ are even but there is an odd circuit $C$ containing $e$ in $G$. Let $C^{\prime}$ be any other circuit in $G$ containing $e$. Then the symmetric difference $\Delta\left(C, C^{\prime}\right)$ is the disjoint union of circuits (this follows from the dual of Theorem 15.2 in [10]) all of which must be even. But cardinality of symmetric difference is mod 2 addition, hence $C^{\prime}$ must also be odd and so in $G$ every circuit containing $e$ is odd while every circuit not containing $e$ is even.

But if $D$ is a circuit in $G / e$, the rank of $D$ in $G / e, r^{\prime \prime}(D)$, equals $|D|-1$ so that in $G, r(D \cup\{e\}) \leq|D \cup\{e\}|-1$ hence $D \cup\{e\}$ is dependent and contains a circuit $D^{\prime}$. If $D^{\prime} \backslash\{e\}$ were properly contained in $D$, then $D$ could not be a minimal dependent set in $G / e$, so that $D$ or $D \cup\{e\}$ is a circuit of $G$. In either case $D$ is an even circuit and $(* *)$ becomes $0=1-1$.

Theorem 10.3. If $G$ is a binary pregeometry and $\chi(G, \lambda)$ is the characteristic polynomial of $G$, then $\chi(G, 2)=1$ if $G$ bas all even circuits and is 0 otherwise.

Proof. Let $\bar{f}(G)=1$ if $G$ has all even circuits and 0 otherwise. Then $(-1)^{r(G)} \bar{f}(G)$ is the Tutte invariant $f(G)$ of (10.2). If $z$ is an isthmus, then $z$ has no circuits and $f(z)=-1$ while if $x$ is a loop, $x$ is an odd circuit and $f(x)=0$. By the fundamental theorem (3.6),

$$
(-1)^{r(G)} \bar{f}(G)=\left.t(G)\right|_{z=-1, x=0}=\left.(-1)^{r(G)} \chi(G, \lambda)\right|_{1-\lambda=-1} .
$$

Corollary 10.4. A spanning subset $S$ of vectors in a vector space $V$ over $G F(2)$ will be distinguished by a unique functional (equivalently will be contained in a unique bond of $L(V)$ or will be disjoint from a unique byperplane) if and only if $S$ bas all even circuits.

Hence a geometry of rank $n$ can be imbedded in the affine space of dimension $n$ over GF(2) if and only if it has no four point lines as minors and no odd circuits.

Proof. This follows from (10.3) and (9.5).

11. Graphic pregeometries. Under certain conditions a pregeometry $G$ has the same point-circuit incidence relationship as the edge-circuit incidence relationship of a multigraph $M$ (graph in which multiple edges and loops are allowed). In that 
case we say the pregeometry is graphic and we may give a special interpretation to the decomposition and many of the invariants of $\$ 4$.

We now list some of the basic definitions and properties of multigraphs and their associated pregeometries.

Definition 11.1. A multigraph $M$ is a function from a set $E$ into the set of unordered pairs $V \& V$ (i.e. $V \times V /\left(v_{i}, v_{j}\right) \sim\left(v_{j}, v_{i}\right)$ ). The set $E$ is called the edges of $M$ and $V$ is called the vertices. The restriction $M^{\prime}=M\left(E^{\prime}\right)$ of $M$ to a subset $E^{\prime} \subseteq E$ is called a submultigraph of $M$.

Denoting the preimage of the diagonal of $V \& V$ by $L(M)$, a graph is a multigraph which is $1-1$ and where $L(M)$ is empty. In particular the underlying graph $\bar{M}$ of a multigraph is the function $M^{\prime} \circ K$ where $M^{\prime}$ is the subgraph on the set $E \backslash L(M)$ and $K$ is the kernel of $M^{\prime}$. Thus exactly one edge is sent to a vertex pair $\left[v_{i}, v_{j}\right]$ iff $i \neq j$ and $M^{-1}([i, j])$ is not empty. $\bar{M}$ can be thought of as a maximal subgraph (submultigraph which is also a graph) of $M$.

A path of length $p$ from a vertex $v$ to a vertex $v^{\prime}$ is a $(p+1)$-tuple of vertices $\left(v=v_{1}, \cdots, v_{p+1}=v^{\prime}\right)$ and $p$-tuple of edges $\left(e_{1}, \cdots, e_{p}\right)$ such that $M\left(e_{i}\right)=\left[v_{i}\right.$, $\left.v_{i+1}\right]$. Path connectedness or connectedness induces the obvious equivalence relation on $V$ and $M$ is connected if $v \sim v^{\prime}$ for all $v, v^{\prime} \in V$. Associated with any subset of edges $E^{\prime}$, we have the equivalence relation on $V$ in which $v_{i} \widetilde{E}^{\prime} v_{j}$ iff $v_{i} \sim v_{j}$ in the submultigraph $M\left(E^{\prime}\right)$.

One may form a pregeometry $G(M)$ whose points are the edges of $M$ and whose closure relation is defined for all $E^{\prime} \subseteq E$ by $\bar{E}^{\prime}=\left\{\left[M^{-1}\left(v_{i}, v_{j}\right)\right]\right\}$ for all $v_{i} \widetilde{E}, v_{j}$. It is easy to verify that this is a geometric closure operator and that the circuits of $G(M)$ are those edges which form a path $\left(v_{1}, \cdots, v_{p+1}\right)$ in which $v_{1}=v_{p+1}$ but no other vertices are equal. The bases of $G(M)$ are maximal sets which contain no circuits, called the spanning trees of a connected multigraph. The one point circuits (loops) of $G$ are the edges in $L(M)$ (the inverse image under $M$ of the diagonal) and the two point circuits of $G$ are those edge pairs which get mapped into the same pair of distinct vertices. Hence if $\bar{M}$ is the graph underlying the multigraph $M$ then $G(\bar{M})$ is the canonical geometry $\bar{G}(M)$ associated with the pregeometry $G(M)$. Also if $E^{\prime} \subseteq E$, then $G\left(M\left(E^{\prime}\right)\right)$ is the subgeometry $G(M(E)) \backslash\left(E \backslash E^{\prime}\right)$.

$M$ is two-connected if $G(M)$ is connected iff $M$ is connected and every two edges lie in some circuit. If $|V|=n$, we define the complete graph $K_{n}$ as a graph in which $\left|M^{-1}\left(\left[v_{i}, v_{j}\right]\right)\right|=1$ for all $v_{i} \neq v_{j}$. Since closed sets of $G(M)$ are in $1-1$ correspondence with partitions of the vertex set into path components, the lattice of closed sets of $K_{n}$ is the lattice of partitions of an $n$-element set. Every geometry of a graph on $n$ vertices is a subgeometry of $K_{n}$.

Proposition 11.2. Let $M$ be a multigraph with associated pregeometry $G$. Then for all $e \in E$, the deletion $G \backslash e$ is the pregeometry of the submultigraph $G(M(E \backslash\{e\}))$; and if $M(e)=\left[v_{i}, v_{j}\right]$ then the contraction $G / e$ is the restriction of 
the composite function $I \circ M$ to $E \backslash\{e\}$ where $I: V \& V \rightarrow V \& V / v_{i} \sim v_{j}$ identifies $\left[v_{i}, v\right]$ and $\left[v_{j}, v\right]$ for all $v \in V$. (G/e can be thought of as coalescing the vertices $v_{i}$ and $v_{j}$ and then removing the loop e.)

If $M_{1}$ and $M_{2}$ are both one-connected multigraphs then the pregeometry $G\left(M_{1} \wedge M_{2}\right)$ is the direct sum $G\left(M_{1}\right) \oplus G\left(M_{2}\right)$ where $M_{1} \wedge M_{2}$ (the wedge product of two multigraphs) is the disjoint union of $M_{1}$ and $M_{2}$ followed by an identification (coalescence) of a vertex $v_{1} \in M_{1}$ and a vertex $v_{2} \in M_{2}$. Conversely, if $M^{\prime}$ is a one-connected multigraph such that $G\left(M^{\prime}\right)=G\left(M_{1}\right) \oplus G\left(M_{2}\right)$ where $M_{1}$ and $M_{2}$ are one-connected then $M^{\prime}=M_{1}^{\prime} \wedge M_{2}^{\prime}$ where $G\left(M_{1}^{\prime}\right)=G\left(M_{1}\right)$ and $G\left(M_{2}^{\prime}\right)=G\left(M_{2}\right)$.

Proof. The M-closure of a set depends only on the vertex partition associated with paths in the set. A subset of $E \backslash\{e\}$ would induce the same vertex partition in $M$ and in $M \backslash e=M(E \backslash\{e\})$ and would thus induce the same closure except that $e$ would not appear in $M \backslash e$. Hence $G(M \backslash e)=G \backslash e$.

Both $G / e$ and $G(M / e)$ are defined on the same edge set, $E \backslash\{e\}$. We will show that the closure operator $J$ in $G / e$ is the same as the closure operator $K$ in $G(M / e)$.

Let $E^{\prime} \subseteq E \backslash\{e\}$ and $e^{\prime} \in E \backslash\{e\}$ be arbitrary such that $M\left(e^{\prime}\right)=\left[v_{k}, v_{m}\right]$ (either or both vertices could be $v_{i}$ or $v_{j}$ ). Then $e^{\prime} \in J\left(E^{\prime}\right)$ iff there is a path in $M$ connecting $v_{k}$ and $v_{m}$ with edges in $E^{\prime} \cup\{e\}$; and $e^{\prime} \in K\left(E^{\prime}\right)$ iff there is a path in $M / e$ connecting $v_{k}$ and $v_{m}$ with edges in $E^{\prime}$. Assume $P$ is a path linking $v_{k}$ and $v_{m}$ in $M$ with edges in $E^{\prime} \cup\{e\}$. Then deleting $e$ wherever it appears in $P$, we get a path in $M / e$ since if $P=\left(\cdots, e_{n}, e, e_{n+1}, \cdots\right)$ then $e_{n}$ and $e_{n+1}$ share the common vertex $\overline{v_{i} v_{j}}$ and $P^{\prime}=\left(\cdots, e_{n}, e_{n+1}, \cdots\right)$ is part of a path in $M / e$.

Conversely, assume $\left(\cdots, e_{n}, e_{n+1}, \cdots\right)$ is a path in $M / e$ in which $e_{n}$ and $e_{n+1}$ share the common vertex $\frac{v_{i} v_{j}}{}$. Then if $e_{n}$ and $e_{n+1}$ share a common vertex in $M\left(\right.$ e.g. $\left.v_{i}\right),\left(\cdots, e_{n}, e_{n+1}, \cdots\right)$ would be part of a path in $M$ while if for example $M\left(e_{n}\right)=\left[v^{\prime}, v_{j}\right]$ and $M\left(e_{n+1}\right)=\left[v_{k}, v^{\prime \prime}\right]$ then $\left(\cdots, e_{n}, e, e_{n+1}, \ldots\right)$ would be part of a path in $M$.

$G\left(M_{1}\right) \oplus G\left(M_{2}\right)$ is the pregeometry formed from the disjoint union of edges and circuits of $M_{1}$ and $M_{2}$. If $M_{1} \wedge M_{2}$ is the wedge product of $M_{1}$ and $M_{2}$ in which $v_{1} \in M_{1}$ and $v_{2} \in M_{2}$ are identified then $M_{1} \wedge M_{2}$ is one-connected since every vertex in $M_{1}$ is joined to $v_{1}$ and every vertex in $M_{2}$ is joined to $v_{2}$. But any closed path containing edges in both $M_{1}$ and $M_{2}$ must list the identified vertex $\overline{v_{1} v_{2}}$ twice. Since $M_{1}$ and $M_{2}$ are subgraphs of $M_{1} \wedge M_{2}, G\left(M_{1}\right)$ and $G\left(M_{2}\right)$ are subgeometries and the circuit set $\underline{C}\left(M_{1} \wedge M_{2}\right)$ is the disjoint union $\underline{C}\left(M_{1}\right) \cup \underline{C}\left(M_{2}\right)$. Also $E\left(M_{1} \wedge M_{2}\right)=E\left(M_{1}\right) \cup E\left(M_{2}\right)$ and hence $G\left(M_{1} \wedge M_{2}\right)=G\left(M_{1}\right) \oplus G\left(M_{2}\right)$.

Conversely, assume $M^{\prime}, M_{1}$ and $M_{2}$ are one-connected and $G\left(M^{\prime}\right)=G\left(M_{1}\right) \oplus$ $G\left(M_{2}\right)$. Then $M^{\prime}$ must have subgraphs $M_{1}^{\prime}$ and $M_{2}^{\prime}$ such that $G\left(M_{i}^{\prime}\right)=G\left(M_{i}\right)(i=1,2)$. If these subgraphs have no vertices in common $M^{\prime}$ is not one-connected (no path can join $v_{1} \in M_{1}^{\prime}$ with $v_{2} \in M_{2}^{\prime}$ ). If they have two vertices $v$ and $v^{\prime}$ in common then since $M_{i}^{\prime}$ is connected there is a minimal path $P_{i}$ in $M_{i}$ joining $v$ and $v^{\prime}(i=1,2)$. 
But then $P_{1} P_{2}$ would contain a circuit intersecting both $M_{1}^{\prime}$ and $M_{2}^{\prime}$ which would contradict the definition of direct sum. Since $E\left(M^{\prime}\right)=E\left(M_{1}^{\prime}\right) \cup E\left(M_{2}^{\prime}\right)$ and $V\left(M^{\prime}\right)=$ $V\left(M_{1}^{\prime}\right) \wedge V\left(M_{2}^{\prime}\right)$ we are done.

Example 11.3. In analogy with the decomposition of $P_{4}^{\prime}$ (the pregeometry of contractions of $K_{4}^{\prime}$ the complete four graph with a multiple edge) in (3.4) and as an illustration of the techniques in (11.2) we will decompose $K_{4}^{\prime}$ in the Tutte subbidecomposition associated with all multigraphs.

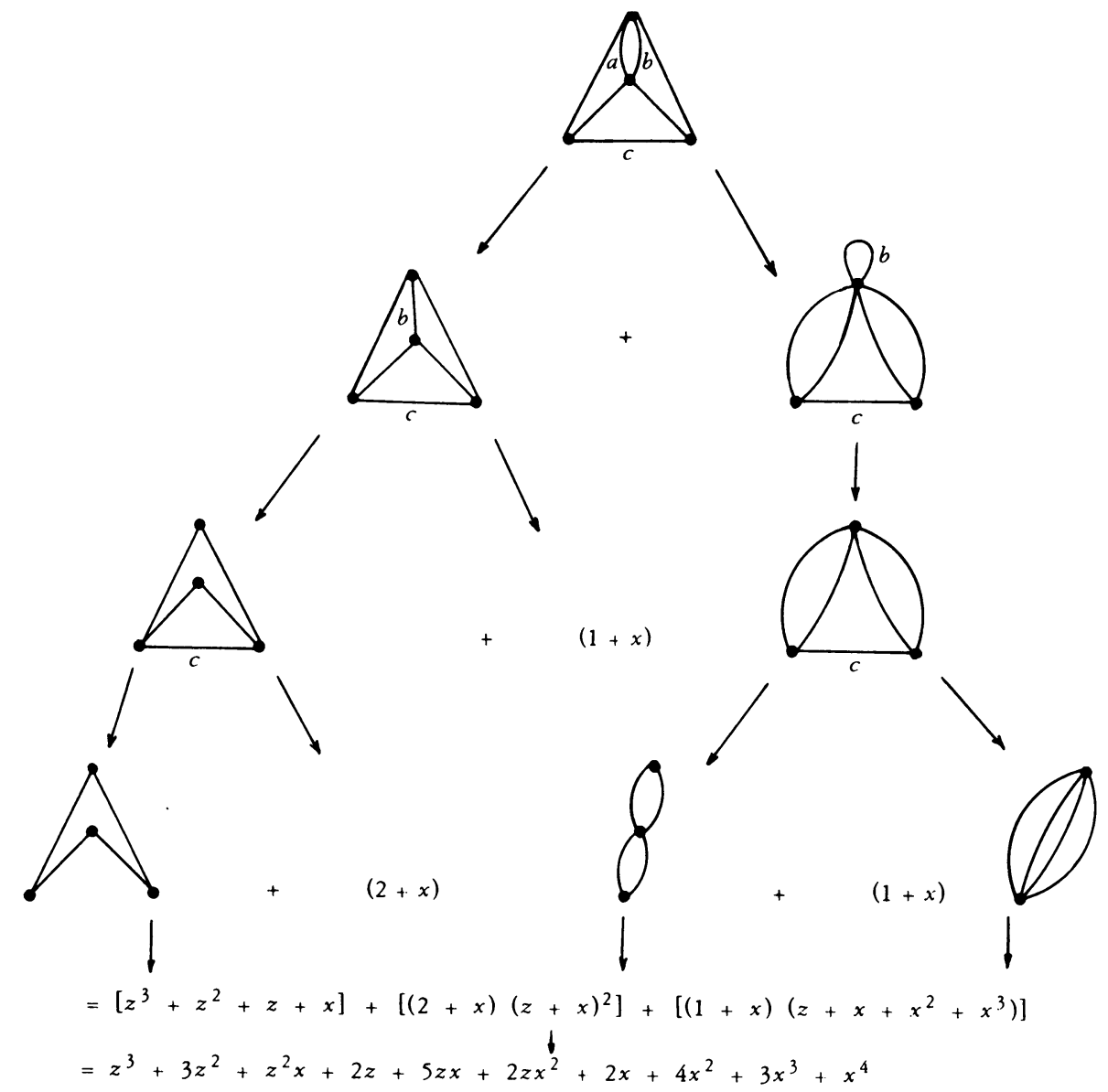

Definition 11.4. A coloring (or $n$-coloring) of a multigraph $M$ is a function $c$ from the vertex set of $M$ to a set (of $n$ elements) such that for all $e \in E, M(e)=$ $\left[v, v^{\prime}\right]$ implies $c(v) \neq c\left(v^{\prime}\right)$. A multigraph is $n$-colorable if it admits an $n$-coloring.

An orientation $\vec{M}$ of $M$ is a function $\vec{M}=O \circ M$ where $O: V \& V \rightarrow V \times V$ assigns to $\left[v_{i}, v_{j}\right]$ either of the ordered pairs $\left(v_{i}, v_{j}\right)$ or $\left(v_{j}, v_{i}\right)$. (We may think of an orientation as an assignment of a direction to each edge such that, for simplicity, we assume each edge joining the same two vertices has the same direction.) 
A chain (or zero-chain) (with values in $A_{n}$ ) on $\vec{M}$ is a function $f$ from $V(\vec{M})$ into an abelian group $A_{n}$ of order $n$. A coboundary (one-coboundary) is a function $\bar{f}$ from $E(\vec{M})$ to $A_{n}$ associated with a chain $f$ such that if $\vec{M}(e)=\left(v_{i}, v_{j}\right)$ then $\bar{f}(e)=$ $f\left(v_{i}\right)-f\left(v_{j}\right)$. It is well known that $\bar{f}$ is a coboundary for some chain iff there exists a subset $\bar{C} \subseteq C$ for every circuit $C$ of $M$ such that $\Sigma_{e \in \bar{C}} \bar{f}(e)=\Sigma_{e \in C \backslash \bar{C}} \bar{f}(e)$. Note also that a coboundary $\bar{f}$ is nowhere zero iff $f$ is a coloring.

Theorem 11.5. Let $M$ be a multigraph with exactly $k$ one-connected components and let $G$ be its associated pregeometry. Then the rank of the pregeometry $r(G)$ equals $|V(M)|-k$; and the number of $\lambda$-colorings of $M, c(M, \lambda)$, equals $\lambda^{k} \chi(G)$.

Proof. The rank of $G(M)$ is the cardinality of a basis (maximal subset of $E(M)$ containing no circuit) or spanning forest $F$. But the intersection of $F$ with any connected component $M_{i}$ of $M$ has cardinality $\left|V\left(M_{i}\right)\right|-1$. Hence $|F|=$ $\sum_{i=1}^{k}\left(\left|V\left(M_{i}\right)\right|-1\right)=|V(M)|-k$.

We will show that $(-1)^{r(M)} c(M, \lambda) / \lambda^{k}$ is an invariant of the decomposition of the Tutte-Grothendieck group and then apply (3.7). If $e \in G$ is neither an isthmus nor a loop then $M \backslash e$ and $M / e$ have the same number of components as $M$. Assume $M(e)=\left[v, v^{\prime}\right]$. The $\lambda$-colorings of $M \backslash e$ may be partitioned into two sets: those in which $f(v) \neq f\left(v^{\prime}\right)$ and those which assign to $v$ and $v^{\prime}$ the same "color". The former set represents all the colorings of $M$ and the latter gives a well defined way to describe all the colorings of $M / e\left(f\left(\overline{v v^{\prime}}\right)=f(\nu)\right)$. Hence $c(M, \lambda)=$ $c(M \backslash e, \lambda)-c(M / e, \lambda)$ and dividing that equation by $(-1)^{r(M)} \lambda^{k}$ we see that $(-1)^{r(M)} c(M, \lambda) / \lambda^{k}$ is a group invariant. We must evaluate it on all pre-Boolean algebras $B_{n m}$. But if $m>0, B_{n m}$ contains a loop and for all $\lambda$ the vertex incident with the loop and hence the pregeometry cannot be colored. If $B_{n_{i}}$ is a one-connected component of $B_{n}$ then we may choose a vertex $v_{1} \in B_{n_{i}}$ and color it any one of $\lambda$ colors. Choose another vertex $v_{2}$ adjacent to it (such that $M^{-1}\left(\left[v_{1}, v_{2}\right]\right.$ ) is nonempty). Then $v_{2}$ may be colored with any color except $f\left(v_{1}\right)$. Choosing recursively any vertex which is incident with vertex chosen before we will have counted $\lambda(\lambda-1)^{|V|-1}=\lambda(\lambda-1)^{|E|}$ colorings for $B_{n_{i}}$. Note that no vertex will be incident to two distinct vertices chosen before since we would then have a circuit. If $B_{n}$ has one-connected components of (edge) cardinality $n_{1}, \cdots, n_{k}$ then since each component may be colored independently we see that $c\left(B_{n}, \lambda\right)=$ $\left(\lambda(\lambda-1)^{n_{1}}\right) \cdots\left(\lambda(\lambda-1)^{n_{k}}\right)=\lambda^{k}(\lambda-1)^{r\left(B_{n}\right)}$. Hence $(-1)^{r(M)} c(M, \lambda) / \lambda^{k}$ is a group invariant with boundary values $(-1)^{n} c\left(B_{n m}, \lambda\right) / \lambda^{k}=(1-\lambda)^{n} \delta(m, 0)$. But $(-1)^{r(G)} \chi(G)$ is a group invariant (in fact it is a ring invariant) with the same values on $B_{n m}$. Hence $\chi(G)=c(M, \lambda) / \lambda^{k}$.

We will apply some of the above techniques to invariants of $K_{n}$, the complete graph on $n$ vertices. 
Proposition 11.6. Let $M=K_{n}\left(a_{1}, \cdots, a_{n-1}\right)$ be the multigraph on the vertex set $v_{1}, \cdots, v_{n}$ where $\left|M^{-1}\left(\left[v_{i}, v_{i}\right]\right)\right|=0$ for all $i,\left|M^{-1}\left(\left[v_{i}, v_{j}\right]\right)\right|=1$ for all $1 \leq$ $i<j \leq n-1$, and $\left|M^{-1}\left(\left[v_{i}, v_{n}\right]\right)\right|=a_{i}$ for all $1 \leq i \leq n-1$. Hence $K_{n}(1,1, \cdots, 1)=$ $K_{n+1}(0, \cdots, 0)=K_{n}$. Let $t_{n}(\cdots)$ be the Tutte polynomial of $K_{n}(\cdots)$. Then $t_{n}$ may be computed from the following recursions:

$t_{n}\left(a_{1}, \ldots, a_{i}, \ldots, a_{n-1}\right)=t_{n}\left(\ldots, a_{i}-1, \ldots\right)$

$$
+x^{a_{i}-1} t_{n-1}\left(a_{1}+1, \ldots, a_{i-1}+1, a_{i+1}+1, \ldots, a_{n-1}+1\right)
$$

for all $a_{i}>0, \Sigma_{j=1}^{n-1} a_{j}>1 ; t_{n}(0, \cdots, 0,1,0, \cdots, 0)=z t_{n-1}(1,1, \cdots, 1) ;$ and $t_{1}=1$.

Proof. We contract and delete edges incident with $v_{n}$. In the first case if $e$ is not an isthmus and $e$ connects $v_{n}$ with $v_{i}$ (where $\left.\left|M^{-1}\left(\left[v_{i}, v_{n}\right]\right)\right|=a_{i}\right)$ its deletion leaves $a_{i}-1$ edges joining $v_{n}$ and $v_{i}$ while its contraction identifies $v_{i}$ and $v_{n}$ and hence creates $a_{i}-1$ loops. For any vertex $v_{j}(j \neq i, n)$, $\left|M^{-1}\left(\left[v_{i}, v_{j}\right]\right)\right|=1$ and $\left|M^{-1}\left(\left[v_{n}, v_{j}\right]\right)\right|=a_{j}$, hence $\left|M^{-1}\left(\left[\frac{j}{v_{i} v_{n}}, v_{j}\right]\right)\right|=a_{j}+1$. The edge $e$ joining $v_{i}$ and $v_{n}$ is an isthmus iff it is the only edge incident with $v_{n}$ in which case $M=e \oplus K_{n-1}$. Finally, $t_{1}=1$ is a boundary condition for the empty graph.

Corollary 11.7.

$$
\begin{aligned}
& \chi\left(K_{n}\right)=(\lambda-1) \ldots(\lambda-n+1), \\
& \mu\left(K_{n}\right)=(-1)^{n-1}(n-1) ! \\
& \beta\left(K_{n}\right)=(n-2) !
\end{aligned}
$$

Proof. All these invariants vanish whenever there is a loop so that $f\left(K_{n}\left(a_{i}, \cdots, a_{n-1}\right)\right)=f\left(K_{n}\left(a_{1}, \cdots, a_{i}-1, \cdots, a_{n-1}\right)\right)$ whenever $a_{i}>1$. Thus for all the invariants above:

$$
f\left(K_{n}\left(a_{1}, \ldots, a_{n-1}\right)\right)=(k-1) f\left(K_{n-1}(1, \ldots, 1)\right)+f\left(K_{n}(0, \ldots, 1, \ldots, 0)\right)
$$

where $k$ is the number of positive $a_{i}$. This recursion for various evaluations of $z$ gives:

$$
(-1)^{n-1} \chi\left(K_{n}\right)=(n-2+(1-\lambda))(-1)^{n-2} \chi\left(K_{n-1}\right)
$$

with initial condition $\chi\left(K_{2}\right)=\lambda-1$ and evaluating this expression at $\lambda=0$ we get the recursion for $\mu$. $\beta\left(K_{n}(0, \cdots, 0,1,0, \cdots, 0)\right)=0$ for $n>2$ so that $\beta\left(K_{n}\right)=$ $(n-2) \beta\left(K_{n-1}\right)$ and $\beta\left(K_{2}\right)=1$.

In [5] Cayley showed that there are $n^{n-2}$ spanning (maximal) trees in the complete graph $K_{n}$. The following corollary suggests a new proof of Cayley's formula. 
Corollary 11.8. If $s_{m}=s_{m}\left(a_{1}, \cdots, a_{n}\right)=\Sigma_{i_{1}}<\cdots i_{m} a_{i_{1}} \cdots a_{i_{m}}$ is the mth degree symmetric polynomial in the variables $a_{1}, \cdots, a_{n}$ and if $\Sigma a_{i}>0$, then the number $c_{n+1}\left(a_{1}, \cdots, a_{n}\right)$ of spanning trees of $K_{n+1}\left(a_{1}, \cdots, a_{n}\right)$ equals $\Sigma_{i=1}^{n} i n^{n-1-i} s_{i}$. In particular, $c_{n+1}(1,0, \cdots, 0)=n^{n-2}$ is the number of spanning trees of $K_{n}$.

Proof. One checks that $c_{i}$ satisfies the recursions of (11.6) when $z=x=1$.

12. Orientable pregeometries. Minty [13] has generalized the notion of orientability (11.4) to unimodular pregeometries (those which may be coordinatized over any field). Crapo [8] has counted the number of nowhere zero coboundaries for such pregeometries.

This enumeration is shown to be a problem properly addressed to an appropriate Tutte subbidecomposition which lends some insight into the structure of orientable pregeometries.

Definition 12.1. The Bond incidence matrix $M_{B}$ of a pregeometry $G$ has its rows indexed by bonds $B_{i} \subseteq G$ and columns indexed by points $p_{j} \in G$ such that

$$
M_{B}\left(B_{i}, p_{j}\right)= \begin{cases}1 & \text { if } p_{j} \in B_{i}, \\ 0 & \text { otherwise. }\end{cases}
$$

We may likewise define the circuit incidence matrix $M_{C}$ of $G$. An orientation of $G$ is a pair of matrices $\left(\vec{M}_{B}, \vec{M}_{C}\right)$ with entries $+1,-1$, and 0 such that $\left|\vec{M}_{B}\right|=$ $M_{B},\left|\vec{M}_{C}\right|=M_{C}$, and $\left(\vec{M}_{B}\right)\left(\vec{M}_{C}\right)^{T}=0$ (i.e. an assignment of either 1 or -1 to the nonzero entries of $M_{B}$ and $M_{C}$ such that their rows become orthogonal). If such an orientation exists, $G$ is called orientable.

Proposition 12.2. Orientability is bereditary. In particular, if $G$ bas orientation $\left(\vec{M}_{B}, \vec{M}_{C}\right)$ then for all nonfactors $p \in G, G / p$ is orientable with orientations $\vec{M}_{B}^{\prime \prime}$ and $\vec{M}_{C}^{\prime \prime}$ where $\vec{M}_{B}^{\prime \prime}$ and $\vec{i}_{C}^{\prime \prime}$ are derivable from the matrices $\vec{M}_{B}$ and $\vec{M}_{C}$ by deleting the pth column and some of the rows. A similar result bolds for $G \backslash p$.

If $G$ is the direct sum of two orientable pregeometries, $G_{1} \oplus G_{2}$, then the pair of matrix direct sums $\left(\vec{M}_{B}\left(G_{1}\right) \oplus \vec{M}_{B}\left(G_{2}\right), \vec{M}_{C}\left(G_{1}\right) \oplus \vec{M}_{C}\left(G_{2}\right)\right)$ is an orientation for $G$.

Finally, if $G$ bas orientation $\left(\vec{M}, \vec{M}^{\prime}\right)$, then $\widetilde{G}$ bas orientation $\left(\vec{M}^{\prime}, \vec{M}\right)$.

Proof. The bonds of $G / p$ are those bonds of $G$ which do not contain $p$. Hence $M_{B}^{\prime \prime}$ is $M_{B}$ with all the rows having a one in the pth position deleted and the $p$ th column deleted.

Let $C$ be a circuit of $G$. If $p \in C$ then $C \backslash\{p\}$ is a circuit of $G / p$. If $p \notin C$ then $C$ is dependent in $G / p$. Conversely, if $C^{\prime}$ is a circuit of $G / p$ then $C^{\prime} \cup\{p\}$ is dependent in $G$. Hence $M_{C}^{\prime \prime}$ is $M_{C}$ with the $p$ th column deleted restricted to rows of minimal support. 
If one restricts $\vec{M}_{B}$ and $\vec{M}_{C}$ to exactly those rows and columns which are retained by $\vec{M}_{B}^{\prime \prime}$ and $\vec{M}_{C}^{\prime \prime}$, then the rows of $\vec{M}_{B}^{\prime \prime}$ are orthogonal to $R$, all rows of $\vec{M}_{C}$ with the pth column deleted (since the rows of $\vec{M}_{B}^{\prime \prime}$ are derived from those rows of $\vec{M}_{B}$ with a 0 in the pth entry). Hence the rows of $\vec{M}_{B}^{\prime \prime}$ are orthogonal to the rows of $\vec{M}_{C}^{\prime \prime}$, a subset of $R$. By duality, identical statements hold for $\left(\vec{M}_{B}^{\prime}, \vec{M}_{C}^{\prime}\right)$, an orientation for $G \backslash p$.

If $G=G_{1} \oplus G_{2}$, then the points, circuits, and bonds of $G$ are the disjoint union of the points, circuits, and bonds of $G_{1}$ and $G_{2}$ respectively. Thus the bond and circuit incidence matrices of $G$ are the respective direct sums and if oriented consistently with the orientations of each factor, the matrix product of the direct sums is the direct sum of the products both of which are zero matrices.

The final statement is a consequence of the fact that a subset $A$ is a circuit of $G$ iff it is a bond of $\widetilde{G}$.

Definition 12.3. Let $G$ be an orientable pregeometry with orientation $\left(\vec{M}_{B}, \vec{M}_{C}\right)$ and let $A_{n}$ be an additively written abelian group of order $n$. A coboundary $f$ of $G$ (with values in $A_{n}$ ) is a function from $G$ to $A_{n}$ associated with a linear combination $\Sigma a_{i} r_{i}$ of the rows of $\vec{M}_{B}$ with coefficients in $A_{n}$. For all $p \in G$ and $f=$ $\Sigma a_{i} r_{i}, f(p)=\Sigma a_{i} r_{i}(p)$ where $a_{i} r_{i}(p)=0, a_{i}$, or $-a_{i}$ if the pth coordinate of $r_{i}$ is 0 , 1 , or -1 respectively. A cycle of $G$ is defined similarly from $\vec{M}_{C}$.

Theorem 12.4. Let $G$ be an orientable pregeometry of rank $n$ and nulity $m$. Then the number $c$ of nowhere zero coboundaries of $G$ (with values in an abelian group of order $k$ ) is given by an evaluation of the characteristic polynomial: $c=$ $(-1)^{n} \chi(G, k)$. The number of nowbere zero cycles equals $\chi(\widetilde{G}, k)=$ $\left.(-1)^{m} t(G)\right|_{z=0, x=1-k}$.

Proof. Let $f(G)$ be the number of nowhere zero coboundaries. We will show that $(-1)^{r(G)} f(G)$ is a Tutte invariant. First note that $f\left(G_{1} \oplus G_{2}\right)=f\left(G_{1}\right) f\left(G_{2}\right)$ since the matrix $\vec{M}_{B}(G)$ is a direct sum so that $f$ is a (nowhere zero) coboundary of $G_{1} \oplus G_{2}$ iff its restriction to $G_{i}$ is a (nowhere zero) coboundary $(i=1,2)$.

We will show that for any nonfactor $p, f(G)=f(G \backslash p)-f(G / p)$ by modifying a proof by Crapo [8]. Let $T$ be a basis for $G$ not containing the point $p$. Then $T$ is a basis for $G \backslash p$. For all $p^{\prime} \in T$ there is a unique row of the matrix $\vec{M}_{B}$ (and $\vec{M}_{B}^{\prime}$ ) whose support (nonzero entries) includes $p^{\prime}$ and otherwise lies in the complement of $T$. Taking arbitrary linear combinations of such rows, we see that coboundaries in $G$ and $G \backslash p$ can take arbitrary values on $T$.

Let $f$ be a coboundary of $G$ and let $f^{\prime}$ be a coboundary of $G \backslash p$ which both agree on the basis $T \subseteq G \backslash p$, and let $p^{\prime \prime} \in(G \backslash p) \backslash T$. If $C$ is the unique circuit in $G \backslash p$ (and hence in $G$ ) which contains $p^{\prime \prime}$ but otherwise lies in $T$, let $r$ and $r^{\prime}$ be the rows in $\vec{M}_{C}$ and $\vec{M}_{C}^{\prime}$ respectively corresponding to $C$. Then $r\left(p_{i}\right)$ can be 
thought of as an endomorphism of $A_{k}$ for all $p_{i} \in G$ where $r\left(p_{i}\right)$ is the $\left(r, p_{i}\right)$ entry of $\vec{M}_{C}$; and $+1(a)=a,-1(a)=-a$, and $0(a)=0$ for all $a \in A_{k}$. Then by orthogonality

$$
0=\sum_{p_{i} \in C} r\left(p_{i}\right) f\left(p_{i}\right)=r\left(p^{\prime \prime}\right) f\left(p^{\prime \prime}\right)+\sum_{p_{i} \in T \cap C} r\left(p_{i}\right) f\left(p_{i}\right) .
$$

Thus $f$ is uniquely determined on $p^{\prime \prime}$ by its values on $T$. But $r\left(p^{\prime \prime}\right)=f^{\prime}\left(p^{\prime \prime}\right) \neq$ 0 and $\Sigma_{p_{i} \in T \cap C} r\left(p_{i}\right) f\left(p_{i}\right)=\Sigma_{p_{i} \in T \cap C^{r}} r^{\prime}\left(p_{i}\right) f^{\prime}\left(p_{i}\right)$, hence $f\left(p^{\prime \prime}\right)=f^{\prime}\left(p^{\prime \prime}\right)$ for all $p^{\prime \prime} \epsilon$ $\{G\} \backslash\{p\}$. But the values $f$ takes on $T$ also determine its value on $p$; hence $f^{\prime}$ is nowhere zero on $G \backslash p$ iff $f$ is nowhere zero on $\{G\} \backslash\{p\}$ (and has some fixed arbitrary value on $p$ ). Thus there is a 1-1 correspondence between nowhere zero coboundaries of $G \backslash p$ and coboundaries of $G$ which are nowhere zero on $\{G\} \backslash\{p\}$.

The rows $R$ of $\vec{M}_{B}^{\prime \prime}$ are exactly those rows $R^{\prime}$ of $\vec{M}_{B}$ with a zero in the pth column (12.2). Since coboundaries of $G$ which are zero on $p$ are freely and uniquely determined by their values on a basis $T(p \in T)$, such coboundaries are linear multiples of $R$ since $T \backslash\{p\}$ is a basis for $G / p$; and so coboundaries can be found taking arbitrary values on $T \backslash\{p\}$ in $G / p$ using rows $R$ which correspond to $R^{\prime}$. Hence there is a 1-1 correspondence between nowhere zero coboundaries of $G / p$ and coboundaries in $G$ which are 0 on $p$ but nonzero elsewhere. Thus $f(G \backslash p)-$ $f(G / p)$ counts the number of coboundaries of $G$ which are nowhere zero on $\{G\} \backslash\{p\}$ and in addition not zero on $p$. But this number is just $f(G)$.

A loop $x$ has no bonds and hence no coboundaries so that $f(x)=0$ while an isthmus has no circuits and hence no constraints so any nonzero element of $A_{k}$ can serve as a coboundary; so $(-1)^{r(z)} f(z)=1-k$ and we are done by the fundamental theorem (3.6). We count the number of cycles by dualizing and noting that cycles of $G$ are coboundaries of $\tilde{G}$ (see 12.2). Then we may apply (4.3) and (4.7).

We remark that the concepts of orientable pregeometries and nowhere zero coboundaries generalize the topological notions of orientation and nowhere zero onecoboundaries (colorings) of multigraphs.

To see this, we orient our multigraph $M$ as in (11.4). Then if $C=\left(v_{1}, e_{1}, v_{2}\right.$, $\left.e_{2}, \cdots, v_{p+1}\right)$ is a circuit and $\vec{M}\left(e_{i}\right)=\left(v_{i}, v_{i+1}\right)$ we place an entry of 1 in the $\left(C, e_{i}\right)$ coordinate of $\vec{M}_{C}$, and we place a -1 if $M\left(e_{i}\right)=\left(v_{i+1}, v_{i}\right)$.

$A$ bond of $M$ consists of all the edges joining two connected blocks $V_{1}$ and $V_{2}$ of a vertex partition of a connected component of $M$. Then if $\vec{M}(e)=\left(\nu, v^{\prime}\right)$ we place an entry of 1 in the $(B, e)$ coordinate of $\vec{M}_{B}$ if $v \in V_{1}$ and $v^{\prime} \in V_{2}$ and a -1 if $v \in V_{2}, v^{\prime} \in V_{1}$. If $r$ is the row of $\vec{M}_{C}$ associated with $C$ and $r$ the row of $\vec{M}_{B}$ associated with $B$ then $\Sigma r\left(e_{i}\right) r^{\prime}\left(e_{i}\right)=0$ since there are an even number of edges in common with $B$ and $C$ and giving those edges $e_{j}$ the induced order from $C$, $r\left(e_{j}\right) r^{\prime}\left(e_{j}\right)$ alternates in sign. 
To lend some insight into the enumerations (11.5) and (12.4) one notes that every chain of $M$ gives rise to a unique coboundary and every coboundary determines all the values of a chain once the value for one vertex has been specified for each one-connected component of $M$. The resulting chain is a coloring iff the coboundary is nowhere zero.

The generalization is meaningful since for example it allows one to consider coboundaries of duals of nonplanar graphs.

\section{BIBLIOGRAPHY}

1. G. D. Birkhoff, A determinant formula for the number of ways of coloring a map, Ann. of Math. (2) 14 (1913), 42-46.

2. T. Brylawski, A combinatorial model for series-parallel networks, Trans. Amer. Math. Soc. 154 (1971), 1-22.

3. - The Möbius function as decomposition invariant, Proc. Waterloo Conference on Möbius Algebras, 1971, pp. 143-148.

4. - The Tutte-Grothendieck ring (to appear).

5. A. Cayley, A theorem on trees, Quart. J. Math. 23 (1889), 376-378.

6. H. Crapo, A higher invariant for matroids, J. Combinatorial Theory 2 (1967), 406417.

7. - Möbius inversion in lattices, Arch. Math. (Basel) 19 (1968), 595-607. MR $39 \# 6791$. $\# 6705$.

8. - _ The Tutte polynomial, Aequationes Math. 3 (1969), 211-229. MR 41 131. MR $33 \# 1240$.

10. H. Crapo and G.-C. Rota, Combinatorial geometries, M. I. T. Press, Cambridge Mass., 1970 (preliminary ed.).

11. J. Hartmanis, Lattice theory of generalized partitions, Canad. J. Math. 11 (1959), 97-106. MR 20 \#6367.

12. D. A. Higgs, Stong maps of geometries, J. Combinatorial Theory 5 (1968), 185-191. MR 38 \#89.

13. G. J. Minty, On the axiomatic foundations of the theories of directed linear graphs, electrical networks and network programming, J. Math. Mech. 15 (1966), 485-520. MR 32 \#5543.

14. G.-C. Rota, On the foundations of combinatorial theory. I. Theory of Möbius functions, Z. Wahrscheinlichkeitstheorie und Verw. Gebiete 2 (1964), 340-368. MR 30 \#4688.

15. - Hedrick lectures, Math. Assoc. of Amer. Annual Meeting, Toronto, 1967. $214-217$.

17. W. T. Tutte, A contribution to the theory of chromatic polynomials, Canad. J. Math. 6 (1954), 80-91. MR 15, 814.

18., A ring in graph theory, Proc. Cambridge Philos. Soc. 43 (1947), 26-40. MR 8, 284 .

19. - Lectures on matroids, J. Res. Nat. Bur. Standards Sect. B 69B (1965), 1 47. MR $31 \# 4023$. 1970.

20. - Introduction to the theory of matroids, American Elsevier, New York,

21. H. Whitney, On the abstract properties of linear dependence, Amer. J. Math 57 (1935), 509-533.

DEPARTMENT OF MATHEMATICS, UNIVERSITY OF NORTH CAROLINA AT CHAPEL HILL, CHAPEL HILL, NORTH CAROLINA 27514 